\title{
Regularity, local behavior and partial uniqueness for self-similar profiles of Smoluchowski's coagulation equation
}

\author{
José A. Cañizo and Stéphane Mischler
}

\begin{abstract}
We consider Smoluchowski's equation with a homogeneous kernel of the form $a(x, y)=x^{\alpha} y^{\beta}+x^{\beta} y^{\alpha}$ with $-1<\alpha \leq \beta<1$ and $\lambda:=\alpha+\beta \in(-1,1)$. We first show that self-similar solutions of this equation are infinitely differentiable and prove sharp results on the behavior of self-similar profiles at $y=0$ in the case $\alpha<0$. We also give some partial uniqueness results for self-similar profiles: in the case $\alpha=0$ we prove that two profiles with the same mass and moment of order $\lambda$ are necessarily equal, while in the case $\alpha<0$ we prove that two profiles with the same moments of order $\alpha$ and $\beta$, and which are asymptotic at $y=0$, are equal. Our methods include a new representation of the coagulation operator, and estimates of its regularity using derivatives of fractional order.
\end{abstract}

\section{Introduction}

\subsection{Smoluchowski's equation}

Smoluchowski's coagulation equation is a well-known model for irreversible aggregation processes. These processes involve a set of particles which can join to form groups of two or more of them, which we call clusters. Below we will briefly present the model; we refer the reader to the reviews $[1,3,10,11]$ for a more detailed background on the equation.

We study this equation in a continuous setting, meaning that the size of a cluster may be any positive number $y \in(0, \infty)$. We are interested in the time evolution of the density of clusters of each possible size, given

2000 Mathematics Subject Classification: 82C21, 45K05, 82C05.

Keywords: Coagulation, self-similarity, regularity, uniqueness, asymptotic behavior. 
by a function $f=f(t, y)$ which depends on the time $t$ and cluster size $y$. The mass of $f$ at a given time $t$ is given by its first moment $\int_{0}^{\infty} y f(t, y) d y$. The continuous Smoluchowski equation reads:

$$
\partial_{t} f(t, y)=C(f(t), f(t))(y)
$$

where

$$
\begin{aligned}
C(f, f)(y):= & \frac{1}{2} \int_{0}^{y} a(z, y-z) f(z) f(y-z) d z \\
& -\int_{0}^{\infty} a(z, y) f(z) f(y) d z,
\end{aligned}
$$

where the coagulation kernel $a=a(x, y)$ is a given nonnegative symmetric function defined on $(0,+\infty) \times(0,+\infty)$, which governs the time rate at which a cluster of size $x$ and cluster of size $y$ aggregate. We write $C(f, f)$ to emphasize that $C$ is a quadratic operator, in agreement with later discussions in which we consider its associated symmetric bilinear form $C(f, g)$.

In many physical models the coagulation kernel $a$ is a homogeneous function [1] in the sense that for some $\lambda \geq 0$ it holds that

$$
a(h x, h y)=h^{\lambda} a(x, y) \quad \text { for } x, y, h>0 .
$$

For our results we will take that as a hypothesis, and in fact we will assume that $a$ is of the following form:

$$
\begin{gathered}
a(x, y):=x^{\alpha} y^{\beta}+x^{\beta} y^{\alpha} \quad \text { for } x, y>0, \\
-1<\alpha \leq \beta<1, \quad \lambda:=\alpha+\beta \in(-1,1) .
\end{gathered}
$$

In some places, we allow $a$ to be a finite linear combination of such terms.

When the kernel is homogeneous, one may look for self-similar solutions, also called scaling solutions: solutions which are given by a rescaling of some fixed function $g$ for all times. In other words, a solution $f=f(t, y)$ to Smoluchowski's equation is self-similar if there exists some nonnegative function $g$ such that

$$
f(t, y)=q(t) g(p(t) y) \quad \text { for all } t, y>0
$$

for some functions $q(t), p(t)>0$. Such a function $g$ is called a self-similar profile (or scaling profile). In this work we will always consider self-similar solutions with finite mass. If $a$ is homogeneous of degree $-1<\lambda<1$, it is known (e.g., [4]) that for $f$ to be a self-similar solution it must happen that

$$
q(t)=\left(t_{0}+t\right)^{-\frac{2}{1-\lambda}} \quad \text { and } \quad p(t)=\left(t_{0}+t\right)^{-\frac{1}{1-\lambda}}
$$


for some constant $t_{0}>0$, and that $g$ satisfies the self-similar profile equation:

$$
2 g+y \partial_{y} g+(1-\lambda) C(g, g)=0 .
$$

The existence of self-similar profiles has been established in $[6,4]$ for a general class of coefficients which in particular cover the case of $a(x, y)$ satisfying (1.3). It is expected (and has been proved in some particular cases) that in general conditions solutions exhibit a universal self-similar behavior in the long time, meaning that they are eventually close to a self similar solution of the equation. The conjecture that this behavior does in fact hold is called the dynamical scaling hypothesis, and a lot of effort has been done to rigorously prove its validity. In particular, it is expected that with "regular" initial conditions (such as compactly supported data) solutions become eventually close to a self-similar solution with finite mass. For the special cases $a(x, y)=1$ or $a(x, y)=x+y$ rather complete results have been proved $[9,12,13]$, but for a general coefficient almost no information is available. It seems likely that further study of the scaling profiles is needed before a more complete understanding of dynamical scaling can be obtained, and our results aim in this direction.

\subsection{Description of the main results}

We first give a proof of the infinite differentiability of self-similar profiles. This fact was already known in the case $\alpha<0$ [4], and here we generalize this result to include the $\alpha \geq 0$ case:

Theorem 1.1. Assume that the coefficient a is of the form (1.3), or is a finite linear combination of terms of that form, all with the same homogeneity degree $\lambda$. Then, any self-similar profile with finite mass for Smoluchowski's equation is infinitely differentiable on $(0, \infty)$.

We remark that in the paper [7], self-similar profiles for $\alpha=0$ were shown to be $\mathcal{C}^{1}$, and in [4, Th. 4.3] solutions for $\alpha \geq 0$ were proved to have a $C^{0, \theta}$ Hölder regularity for any $0 \leq \theta<1-\lambda$.

Regarding the asymptotic behavior of scaling profiles at 0 and $\infty$, some estimates have been rigorously proved $[6,5,4]$, and are consistent with the very complete previous formal calculations by van Dongen and Ernst [17]. Exponential decay of solutions as $y \rightarrow \infty$ was proved in $[4,7]$, but the rate of decay is still far from the rate expected by formal calculations in [17]. As for the behavior at $y=0$, one has to separately treat the $\alpha=0, \alpha>0$ and $\alpha<0$ cases:

1. For $\alpha=0$, a very precise result was given by Fournier and Laurençot in [7], making rigorous the conclusions in [17]: a self-similar solution 
is asymptotic to a constant times $y^{-\tau}$ as $y \rightarrow 0$, for some $1<\tau<$ $\min \{3 / 2,1+\lambda\}$. An intriguing property of this result is that in order to know the numerical value of $\tau$ one needs to compute the solution itself, as $\tau$ is given in terms of the $\lambda$-moment of the solution.

2. For $\alpha>0$, the formal arguments in [17] suggest that a scaling profile $g$ should be asymptotic to a constant times $y^{-1-\lambda}$ when $y \rightarrow 0$. So far, it has been rigorously proved that all the moments of order $\sigma>\lambda$ of $g$ are finite, while all moments of order $\sigma<\lambda$ are infinite.

3. For $\alpha<0$, exponential decay of solutions at $y=0$ was proved in [4]. Our contribution here is a refinement of this behavior which coincides with the formal result in [17], and which is needed in the proof of uniqueness for $\alpha<0$.

Our precise result for $\alpha<0$ is the following:

Theorem 1.2. Assume that the coagulation kernel is of the form (1.3) with $\alpha<0$. Then, if $g$ is a (nonzero) self-similar profile for Smoluchowski's coagulation equation, it holds that

$$
g(y) \sim K_{0} e^{-\Lambda(y)} \quad \text { as } y \rightarrow 0
$$

for some strictly positive constant $K_{0}$, where

$$
\Lambda(y):=2 \log y-(1-\lambda)\left(\frac{M_{\beta}}{\alpha} y^{\alpha}+\frac{M_{\alpha}}{\beta} y^{\beta}\right),
$$

and $M_{\alpha}, M_{\beta}$ are the moments of order $\alpha$ and $\beta$ of $g$. In addition, the function $y \mapsto g(y) e^{\Lambda(y)}$ is decreasing on $(0, \infty)$.

Remark 1.3. Notice that in the case $\beta>0$ the term in $y^{\beta}$ inside $\Lambda$ does not play any role in the behavior of $g$ as $y \rightarrow 0$.

Remark 1.4. The constant $K_{0}$ that appears here is just the one related to the asymptotic behavior of a particular self-similar profile $g$; in particular, it may be different for different self-similar profiles.

We do not prove the asymptotic behavior of self-similar profiles at $y=0$ in the case $\alpha=0$ because this was already proved in [7]. However, the same techniques employed in this paper, in particular the rewriting of the equation in Section 5, may be used to give a proof of it which is somewhat different from the one in [7]. Lemma 5.4 below easily implies the following additional information on a profile $g$ : 
Theorem 1.5. Assume that the coagulation kernel is of the form (1.3) with $\alpha=0$. If $g$ is a self-similar profile for Smoluchowski's coagulation equation, then the function $y \mapsto y^{\tau-1} \int_{y}^{\infty} g(z) d z$ is decreasing on $(0, \infty)$, where

$$
\tau:=2-(1-\lambda) M_{\lambda}[g] \text {. }
$$

We also prove the following partial uniqueness results:

Theorem 1.6. Consider a coagulation kernel of the form (1.3) with $\alpha=0$. Assume that $g_{1}$ and $g_{2}$ are two self-similar profiles of the same mass for Smoluchowski's equation, and also that $M_{\lambda}\left[g_{1}\right]=M_{\lambda}\left[g_{2}\right]$. Then, $g_{1}=g_{2}$.

Here and in the rest of the paper we use the notation $M_{\mu}[g]$ to denote the $\mu$-moment of a function $g$, for any $\mu \in \mathbb{R}$ :

$$
M_{\mu}[g]:=\int_{0}^{\infty} y^{\mu} g(y) d y .
$$

For coefficients with $\alpha<0$ our result is:

Theorem 1.7. Consider a coagulation kernel of the form (1.3) with $\alpha<0$. Assume that $g_{1}$ and $g_{2}$ are two self-similar profiles for Smoluchowski's equation such that

$$
\begin{aligned}
M_{\alpha}\left[g_{1}\right] & =M_{\alpha}\left[g_{2}\right]=: M_{\alpha}, \\
M_{\beta}\left[g_{1}\right] & =M_{\beta}\left[g_{2}\right]=: M_{\beta}, \\
\lim _{y \rightarrow 0} g_{1}(y) e^{\Lambda(y)} & =\lim _{y \rightarrow 0} g_{2}(y) e^{\Lambda(y)},
\end{aligned}
$$

where

$$
\Lambda(y):=2 \log y-(1-\lambda)\left(\frac{M_{\beta}}{\alpha} y^{\alpha}+\frac{M_{\alpha}}{\beta} y^{\beta}\right) .
$$

Then, $g_{1}=g_{2}$.

Remark 1.8. The limit at $y=0$ which appears in this result is proved to exist in Section 6; see Theorem 1.2 below. Using it it is easy to see that condition (1.9) in the above theorem is equivalent to the requirement that $g_{1} \sim g_{2}$ when $y \rightarrow 0$, this is, $\lim _{y \rightarrow 0} g_{1}(y) / g_{2}(y)=1$.

To our knowledge, no uniqueness result at all was available for self-similar profiles of Smoluchowski's equation. The natural conjecture is that the full uniqueness result should hold, this is, that two scaling profiles with the same mass are necessarily equal. This does hold in the better understood cases with $a(x, y)=1$ and $a(x, y)=x+y$, but the techniques we use here do not seem enough to show this for general $\lambda$. A central difficulty is the fact that the moments of the equation $\left(M_{\lambda}\right.$ when $\alpha=0$, or $M_{\alpha}, M_{\beta}$ when $\left.\alpha<0\right)$ are a global property of the solution that cannot be computed a priori (unlike the $a(x, y)=1$ or $a(x, y)=x+y$ cases), and which are even not easy to approximate numerically [2]. 


\subsection{Tools and method of proof}

Among the tools used to prove the above results we highlight a new representation of the coagulation operator, which is given in Section 3, and a rewriting of the self-similar profile equation, given in Section 5. Let us comment on them briefly.

In order to rewrite the coagulation operator, define a distribution associated to any function $f$ of finite mass. We use the Banach space $L_{1}^{1}$ of real measurable functions on $(0, \infty)$ with finite first moment:

$$
L_{1}^{1}:=L^{1}((0, \infty) ; y d y) .
$$

In general, we use the notation

$$
L_{k}^{1}:=L^{1}\left((0, \infty) ; y^{k} d y\right)
$$

for $k \in \mathbb{R}$.

Definition 1.9. (Distribution associated to $f \in L_{1}^{1}$ ). Given a function $f \in L_{1}^{1}$, we define the distribution $\{f\}$ on $\mathbb{R}$ as

$$
\langle\{f\}, \phi\rangle:=\int_{0}^{\infty} f(z)(\phi(z)-\phi(0)) d z \quad \text { for } \phi \in \mathcal{C}_{0}^{\infty}(\mathbb{R}) .
$$

Note that when $f$ is not absolutely integrable at 0 , this is just the classical definition of the finite part of the integral $\int_{0}^{\infty} \phi(y) f(y) d y$ [16]. Here, we keep the same expression even when $f$ is integrable. Then, for a coagulation kernel of the form (1.3), the coagulation operator may be written as

$$
C(g, g)=\left\{y^{\alpha} g\right\} *\left\{y^{\beta} g\right\} .
$$

Here we are considering $C(g, g)$ defined as a distribution; for a more precise statement, see Section 3.

The above expression has the advantage of being simple and lending itself to convenient and perfectly rigorous manipulations. For example, it is easy to recover the known expression for the primitive of $C(g, g)$, as the convolution above commutes with integration and derivation operators. Furthermore, it gives some insight into the way $C(g, g)$ works: if we are working, say, in the case $\alpha=0$, where $g(y) \sim K y^{-\tau}$ when $y \rightarrow 0$ for some $K>0$, one can see that $C(g, g)$ is in many respects analogous to a fractional derivative of $g$ of order $\tau-1$, as a fractional derivative of this order is precisely the convolution with $\left\{y^{-\tau}\right\}$, times a constant. This is crucially used in the proof of the differentiability of profiles in Section 4, where we carry out a bootstrap argument which shows that if a profile $g$ is $k$ times differentiable, then the self-similar profile equation implies that it must be in fact $k+1-\lambda$ times differentiable. The use of fractional derivatives comes naturally in this context, and actually a different version of this bootstrap argument has 
already been used in [7] to show that profiles for $\alpha=0$ are $\mathcal{C}^{1}$. There, it was necessary to first show a certain Hölder regularity of the solution, and then use this information to obtain $\mathcal{C}^{1}$ regularity. Formulating this in terms of gain of fractional derivatives makes it easy to iterate the argument to obtain $\mathcal{C}^{\infty}$ regularity and extend it to other kernels.

For the study of the behavior of solutions near $y=0$ and the proof of uniqueness we rewrite the self-similar profile equation by solving the differential part, along with any other term which can be separated and solved; see Section 5 for a statement of this.

Finally, let us sketch the idea of the proof of our uniqueness result. A fundamental obstacle that makes equation (1.4) difficult to study is the fact that it involves the nonlocal term $C(g, g)$, which makes it very different from an ordinary differential equation. However, the coagulation operator for a constant coefficient has a gain part which only uses values of the function $g$ less than $y$, and a loss part which is nonlocal only through the appearance of the integral of $g$. The idea is then to solve the latter part of the equation, which is simpler, assuming the value of the involved moments is given, and then look at the remaining part as an equation which is local near 0, to which the kind of arguments used in the theory of o.d.e.s can be adapted.

This idea works well for coefficients with $\alpha<0$, as then the solution decays rapidly near $y=0$, but it is not directly applicable for coefficients with $\alpha=0$, as then solutions are known not to be integrable near 0 and the gain and loss parts cannot be separated in the same way (one cannot separate the integral of $g$, as this term does not make sense). But, one can still find a way to separate the equation for the primitive of $g$ in a similar way, and then carry out the argument on it. This line of reasoning is followed in Section 7, where Theorems 1.6 and 1.7 are proved. This idea depends crucially on the fact that in the case $\alpha \leq 0$ the self-similar solution behaves better than $y^{-1-\lambda}$ near $y=0$, so that the linear operator $L_{g}(f):=C(f, g)$ is regularizing near $y=0$. Hence, this method does not give new information in the case $\alpha>0$, where solutions are expected to be asymptotic to $y^{-1-\lambda}[17]$.

\subsection{Organization of the paper}

In the next section we give the basic definitions of self-similar profile, and precisely define the coagulation operator as a distribution on $(0, \infty)$. In Section 3 we give the representation of this operator in the way mentioned above, which in particular extends it naturally to a distribution on $(0, \infty)$. This result is a central idea in the proof of infinite differentiability of scaling profiles, to which Section 4 is devoted. Section 5 proves that the self-similar profile equation can be rewritten, as we briefly explained before, in a way 
which is very useful to prove the asymptotic behavior of $\alpha<0$ profiles at $y=0$ (given in Section 6). Finally, we prove our uniqueness Theorems 1.6 and 1.7 in Section 7.

In an appendix (Section 8) we include a very brief introduction to fractional derivatives in order to clarify the notation and definitions we are using, as they are not completely standard in the literature. We also prove some simple but delicate results on fractional differentiation which are used in this paper, and for which we could not find a reference which gives the explicit statement.

\section{Preliminaries: self-similar profiles}

When one wants to define precisely the concept of self-similar profile, it is a well-known inconvenience that in order for $C(g, g)$ to be well defined by expression (1.2), $g$ must meet certain conditions; the ones which are usually imposed are finiteness conditions on certain moments near 0 and $\infty$, which are not satisfied by the natural solutions of (1.4), known to have a nonintegrable singularity at $y=0[7,17,4,6]$. Hence, the definition of $C(g, g)$ is often changed for a less restrictive weak formulation by integrating against a suitably regular test function $\phi$ with compact support on $(0, \infty)$, which we will do next. Also, $C(g, g)$ is quadratic in $g$, and we will need to consider its associated symmetric bilinear operator, so we actually give a weak definition for the latter:

Definition 2.1. Let $a$ be a symmetric nonnegative measurable function defined on $(0, \infty) \times(0, \infty)$, and $f, g$ be locally integrable functions defined on $(0, \infty)$ such that

$$
\int_{0}^{\infty} \int_{0}^{\infty} a(y, z) y z|f(y) g(z)| d y d z<\infty .
$$

We define the coagulation operator $C(f, g)$ associated to the coagulation coefficient $a$ as the following distribution on $(0, \infty)$ :

$$
\begin{array}{r}
\langle C(f, g), \phi\rangle=\frac{1}{2} \int_{0}^{\infty} \int_{0}^{\infty} a(y, z) f(y) g(z)(\phi(y+z)-\phi(y)-\phi(z)) d x d z \\
\text { for } \phi \in \mathcal{C}_{0}^{\infty}(0, \infty) .
\end{array}
$$

Remark 2.2. For $\phi \in \mathcal{C}_{0}^{\infty}(0, \infty)$ there is always a constant $M>0$ (which depends on the compact support of $\phi$ ) such that

$$
|\phi(y+z)-\phi(y)-\phi(z)| \leq C y z, \quad \text { for } y, z>0,
$$

which can be readily seen by the Mean Value Theorem. Then, the integral (2.2) is finite by (2.1). 
When $f$ is regular enough, $\langle C(f, f), \phi\rangle$ can be see to be equal to the integral $\int_{0}^{\infty} \phi(y) C(f, f)(y) d y$, where $C(f, f)(y)$ is given by expression (1.2). Condition (2.1) can be somewhat weakened in some cases by loosening the integrability condition on $f$ at $+\infty$, but the above one is simpler and will be enough for our purposes.

Definition 2.3. (Self-similar profile). Assume that the coagulation coefficient $a$ is homogeneous of degree $\lambda$. A nonnegative locally integrable function $g:(0, \infty) \rightarrow[0, \infty)$ for which

$$
\begin{gathered}
\int_{0}^{\infty} y g(y) d y<\infty \\
\int_{0}^{\infty} \int_{0}^{\infty} a(y, z) y z|g(y) g(z)| d y d z<\infty
\end{gathered}
$$

is a self-similar profile for Smoluchowski's coagulation equation if equation (1.4) holds in the sense of distributions on $(0, \infty)$; this is, if

$$
2 g+y \partial_{y} g+(1-\lambda) C(g, g)=0 .
$$

\section{A representation of the coagulation operator}

In this section we want to give a representation of the coagulation operator when $a$ has the form (1.3) which sheds some light on its structure, and in particular will be very helpful to prove our regularity results later. To begin with, let us give a natural extension of $C(f, g)$ from Definition 2.1 to a distribution on $\mathbb{R}$ :

Definition 3.1. Take a coagulation coefficient $a$ and functions $f, g$ in the conditions of Definition 2.1. We define the coagulation operator associated to the coagulation coefficient $a$, applied to $f, g$, as the distribution $C(f, g)$ on $\mathbb{R}$ given by

$$
\begin{array}{r}
\langle C(f, g), \phi\rangle:=\frac{1}{2} \int_{0}^{\infty} \int_{0}^{\infty} a(x, z) f(x) g(z)(\phi(x+z)-\phi(x)-\phi(z)+\phi(0)) d x d z \\
\text { for all } \phi \in \mathcal{C}_{0}^{\infty}(\mathbb{R}) .
\end{array}
$$

It is easy to see that this is well defined as a distribution on $\mathbb{R}$, even with the weak requirements on $f$ and $g$, and that it extends Definition 2.1. Note the addition of the term $\phi(0)$, which does not make a difference when $\phi$ has compact support on $(0, \infty)$; later we will see how this extension comes about naturally. To give our representation for $C(f, g)$ we will use the notation from Definition 1.9. Let us initially treat the case of a constant coefficient $a \equiv 1$, and then see how it extends to other coefficients: 
Proposition 3.2. Take $f, g \in L_{1}^{1}$, and let $C_{0}$ be the coagulation operator with a constant coefficient $a \equiv 1$ as given in Definition 3.1. Then,

$$
C_{0}(f, g)=\frac{1}{2}\{f\} *\{g\} \quad \text { as distributions on } \mathbb{R} \text {. }
$$

Here, the convolution $\{f\} *\{g\}$ is understood as a convolution of distributions with compact support to the left [16]. This expression is surprisingly simple, and is well-suited for the study of the coagulation operator when the functions $f, g$ have a singularity at 0 , which is the case with some self-similar profiles. Its proof just consists of writing out the definitions:

Proof. For $\phi \in \mathcal{C}_{0}^{\infty}(\mathbb{R})$,

$$
\langle\{f\} *\{g\}, \phi\rangle=\langle\{f\},(\mathcal{R}\{g\}) * \phi\rangle,
$$

(where $\mathcal{R}$ is the reflection operator, $\mathcal{R} \phi(y):=\phi(-y)$, defined by duality on distributions) and

$$
((\mathcal{R}\{g\}) * \phi)(x)=\left\langle\{g\}, \tau_{-x} \phi\right\rangle=\int_{0}^{\infty} g(z)(\phi(x+z)-\phi(x)) d z,
$$

so from (3.2) we have

$$
\begin{aligned}
\langle\{f\} *\{g\}, \phi\rangle= & \int_{0}^{\infty} f(x)(((\mathcal{R}\{g\}) * \phi)(x)-((\mathcal{R}\{g\}) * \phi)(0)) d x \\
= & \int_{0}^{\infty} f(x)\left(\int_{0}^{\infty} g(z)(\phi(x+z)-\phi(x)) d z\right. \\
& \left.-\int_{0}^{\infty} g(z)(\phi(z)-\phi(0)) d z\right) d x \\
= & \int_{0}^{\infty} \int_{0}^{\infty} f(x) g(z)(\phi(x+z)-\phi(x)-\phi(z)+\phi(0)) d x d z
\end{aligned}
$$

which is our result, in view of expression (3.1).

This directly gives a representation of the coagulation operator $C(f, g)$ with a coagulation kernel $a$ satisfying (1.3), as the following relation holds for any $f, g$ satisfying (2.1):

$$
C(f, g)=C_{0}\left(y^{\alpha} f, y^{\beta} g\right)+C_{0}\left(y^{\beta} f, y^{\alpha} g\right),
$$

where $C(f, g)$ is the operator associated to $a$, and the equality is an equality of distributions on $\mathbb{R}$. 
Theorem 3.3. Assume that the coagulation operator a is of the form (1.3), and take $f, g$ which satisfy (2.1). Then, the coagulation operator associated to a (as given in Definition 3.1) can be written as

$$
C(f, g)=\frac{1}{2}\left(\left\{y^{\alpha} f\right\} *\left\{y^{\beta} g\right\}+\left\{y^{\beta} f\right\} *\left\{y^{\alpha} g\right\}\right),
$$

where equality holds as distributions on $\mathbb{R}$.

We emphasize that this operator is defined as a distribution on $\mathbb{R}$ and, as pointed out after Definition 3.1, extends the usual operator $C$ from Definition 2.1 (which is a distribution on $(0, \infty)$ ).

\section{Local regularity}

With the representation of the coagulation operator $C(g, g)$ given in Theorem 3.3, the study of its regularity can be viewed as the study of the regularity of convolutions of the above type, which is a more manageable problem. The difficulty here is that when $\alpha \geq 0$ a scaling solution $g$ is not integrable near 0 , but is expected to be very regular locally, and hence we need to study the convolution of functions which have singularities at 0 . Precisely, the following general result on the local integrability of scaling profiles near $y=0$ is known (see [6] or [4] for a proof):

Proposition 4.1. Assume that the coagulation coefficient $a$ is of the form (1.3), or is a finite linear combination of terms of that form, all with the same homogeneity degree $\lambda$. Then, all self-similar profiles $g$ for Smoluchowski's equation (in the sense of Definition 2.3) satisfy that

$$
\int_{0}^{R} y^{k} g(y) d y<\infty \quad \text { for all } R>0 \text { and all } k>\lambda .
$$

For convenience, we will measure the regularity of a function by looking at how many of its derivatives are locally integrable on $(0, \infty)$. To study the regularity of $C$ we will need to use an interesting relationship between the kind of singularity of a function near 0 and the local integrability of its fractional integrals, which we give in lemma 4.3 below. Let us start with the following elementary lemma that we state without proof:

Lemma 4.2. For $0<k<1$,

$$
\int_{0}^{\infty}\left(x^{k-1}-(z+x)^{k-1}\right) d x=\frac{1}{k} z^{k} \quad \text { for } z>0 .
$$


Lemma 4.3. If $f \in L_{k}^{1}$, with $0<k \leq 1$, then

$$
\left\|D^{-k}\{f\}\right\|_{L^{1}(\mathbb{R})} \leq \frac{2}{\Gamma(k+1)}\left\|y^{k} f\right\|_{L^{1}(0, \infty)} .
$$

Remark 4.4. We recall that we denote $L_{k}^{1}:=L^{1}\left((0, \infty) ; y^{k} d y\right)$ as in equation (1.11).

Proof. For any $\phi \in \mathcal{C}_{0}^{\infty}(\mathbb{R})$ we will prove that

$$
\left|\left\langle D^{-k}\{f\}, \phi\right\rangle\right| \leq \frac{2}{\Gamma(k+1)}\left\|y^{k} f\right\|_{L^{1}(0, \infty)}\|\phi\|_{\infty},
$$

which is equivalent to our inequality. We have:

$$
\left\langle D^{-k}\{f\}, \phi\right\rangle=\left\langle\{f\}, D_{-k} \phi\right\rangle=\int_{0}^{\infty} f(z)\left(D_{-k} \phi(z)-D_{-k} \phi(0)\right) d z,
$$

and the part inside the parentheses is

$$
\begin{aligned}
& D_{-k} \phi(z)-D_{-k} \phi(0)= \\
& \quad=\frac{1}{\Gamma(k)} \int_{z}^{\infty} \phi(x)(x-z)^{k-1} d x-\frac{1}{\Gamma(k)} \int_{0}^{\infty} \phi(x) x^{k-1} d x \\
& \quad=\frac{1}{\Gamma(k)} \int_{z}^{\infty} \phi(x)\left((x-z)^{k-1}-x^{k-1}\right) d x-\frac{1}{\Gamma(k)} \int_{0}^{z} \phi(x) x^{k-1} d x .
\end{aligned}
$$

We put back this these two terms in (4.3), and bound them separately. The first term is 0 when $k=1$, and for $0<k<1$ we have

$$
\begin{aligned}
\int_{0}^{\infty}|f(z)| & \int_{z}^{\infty}|\phi(x)|\left|(x-z)^{k-1}-x^{k-1}\right| d x d z \leq \\
& \leq\|\phi\|_{\infty} \int_{0}^{\infty}|f(z)| \int_{z}^{\infty}\left|(x-z)^{k-1}-x^{k-1}\right| d x d z \\
& =\frac{1}{k}\|\phi\|_{\infty} \int_{0}^{\infty} z^{k}|f(z)| d z
\end{aligned}
$$

thanks to Lemma 4.2. As for the second term in (4.4), putting it into (4.3) we have

$$
\begin{aligned}
\int_{0}^{\infty}|f(z)| \int_{0}^{z}|\phi(x)| x^{k-1} d x d z & \leq\|\phi\|_{\infty} \int_{0}^{\infty}|f(z)| \int_{0}^{z} x^{k-1} d x d z \\
& =\frac{1}{k}\|\phi\|_{\infty} \int_{0}^{\infty} z^{k}|f(z)| d z
\end{aligned}
$$


Then, equations (4.3)-(4.6) prove that

$$
\left|\left\langle D^{-k}\{f\}, \phi\right\rangle\right| \leq \frac{2}{k \Gamma(k)}\|\phi\|_{\infty} \int_{0}^{\infty} z^{k}|f(z)| d z,
$$

which proves inequality (4.2), taking into account that $k \Gamma(k)=\Gamma(k+1)$.

In the light of the above lemma, our next result can be understood as saying: if two functions are locally regular but have a nonintegrable singularity at 0 , their convolution is slightly less regular. How much less regular it is depends on the nature of the singularity. In particular, the local regularity of the convolution depends only on local properties of the initial functions, which is a general property of the convolution operation. In the next lemma, the reader can keep in mind that $\mu$ will be negative when we use it, so a function $f$ for which $D^{\mu} f$ is integrable may well be not integrable near 0, as Lemma 4.3 makes clear.

Lemma 4.5. Let $T, S$ be two distributions on $\mathbb{R}$ with support on $[0, \infty)$ (this is, $T, S \in \mathcal{D}_{L}^{\prime}$ ), and assume that

1. For some $\nu \in \mathbb{R}, D^{\nu} T$ and $D^{\nu} S$ are locally integrable on $(0, \infty)$.

2. For some $\mu \leq \nu, D^{\mu} T, D^{\mu} S$ are locally integrable on $\mathbb{R}$.

Then, the distribution $D^{\mu+\nu}(T * S)$ is locally integrable on $(0, \infty)$.

Proof. We break $T$ and $S$ into a part near 0 , an intermediate part, and a part near $\infty$. For this, choose $0<\epsilon<1 / 4$. We can find smooth nonnegative cutoff functions $\Phi_{0}, \Phi_{1}, \Phi_{2}$ on $(0, \infty)$ such that

$$
\begin{array}{ll}
\Phi_{0} \equiv 1 \text { on }(0, \epsilon), & \Phi_{0} \equiv 0 \text { on }(2 \epsilon, \infty) \\
\Phi_{1} \equiv 1 \text { on }\left(2 \epsilon, \frac{1}{\epsilon}\right), & \Phi_{1} \equiv 0 \text { on }(0, \epsilon) \cup\left(\frac{2}{\epsilon}, \infty\right) \\
\Phi_{2} \equiv 1 \text { on }\left(\frac{2}{\epsilon}, \infty\right), & \Phi_{2} \equiv 0 \text { on }\left(0, \frac{1}{\epsilon}\right)
\end{array}
$$

and such that

$$
\Phi_{0}+\Phi_{1}+\Phi_{2} \equiv 1 \text { on }(0,+\infty) .
$$

In other words, $\Phi_{0}, \Phi_{1}, \Phi_{2}$ form a partition of unity on $(0, \infty)$ subordinated to the open cover $(0,2 \epsilon) \cup(\epsilon, 2 / \epsilon) \cup(1 / \epsilon, \infty)$. Then,

$$
\begin{aligned}
& S=S \Phi_{0}+S \Phi_{1}+S \Phi_{2}=: S_{0}+S_{1}+S_{2}, \\
& T=T \Phi_{0}+T \Phi_{1}+T \Phi_{2}=: T_{0}+T_{1}+T_{2},
\end{aligned}
$$

where we have denoted $S_{i}:=S \Phi_{i}, T_{i}:=T \Phi_{i}$ for $i=0,1,2$. We can break the convolution $S * T$ by using this decomposition. Note that for $i=0,1,2$, 
$S_{2} * T_{i}$ is zero on $(0,1 / \epsilon)$, and the same happens with $S, T$ interchanged; as we are only interested in studying the regularity of $S * T$ on a bounded interval, we can disregard these terms and write

$$
S * T=S_{0} * T_{0}+S_{0} * T_{1}+S_{1} * T_{0}+S_{1} * T_{1} \quad \text { on }(0,1 / \epsilon) .
$$

Similarly, the term $S_{0} * T_{0}$ is zero on $(4 \epsilon, \infty)$, so we have

$$
S * T=S_{0} * T_{1}+S_{1} * T_{0}+S_{1} * T_{1} \quad \text { on }(4 \epsilon, 1 / \epsilon) .
$$

Then, we can write $D^{\mu+\nu}$ of each of these terms by using Theorem 8.8:

$$
\begin{aligned}
& D^{\mu+\nu}\left(S_{0} * T_{1}\right)=\left(D^{\mu} S_{0}\right) *\left(D^{\nu} T_{1}\right) \\
& D^{\mu+\nu}\left(S_{1} * T_{0}\right)=\left(D^{\nu} S_{1}\right) *\left(D^{\mu} T_{0}\right) \\
& D^{\mu+\nu}\left(S_{1} * T_{1}\right)=\left(D^{\mu} S_{1}\right) *\left(D^{\nu} T_{1}\right) .
\end{aligned}
$$

By the hypotheses of the lemma, we can see that all of the terms that take part in the convolutions on the right hand side are integrable functions, as the product by $\Phi_{0}$ or $\Phi_{1}$ does not change their local regularity properties (Theorem 8.10). Let us do the reasoning for $D^{\mu} S_{1}$ : as $D^{\nu} S$ is integrable on $(4 \epsilon, 1 / \epsilon)$ by hypothesis, we have that $D^{\nu}\left(S \Phi_{1}\right)=D^{\nu} S_{1}$ is integrable on that interval by Theorem 8.10; then, thanks to Lemma 8.11, $D^{\mu} S_{1}$ is also, as $\mu \leq \nu$. The rest of the terms can be treated analogously, and are seen to be integrable without the help of Lemma 8.11.

Then, all the terms on the right hand side of (4.8)-(4.10) are convolutions of integrable functions, and hence are integrable, and (4.7) then proves that $D^{\mu+\nu}(S * T)$ is integrable on $(4 \epsilon, 1 / \epsilon)$.

Proposition 4.6. Take a coagulation coefficient a which is of the form (1.3) with $\alpha \geq 0$, and $C$ the coagulation operator associated to a (given by Definition 3.1). Assume that $g:(0, \infty) \rightarrow \mathbb{R}$ is such that

1. $y^{k} g$ is locally integrable on $[0, \infty)$ for some $1 \geq k \geq \beta$,

2. and $D^{\nu} g$ is locally integrable on $(0, \infty)$ for some $\nu \geq 0$.

Then, $D^{\alpha-k+\nu} C(g, g)$ is locally integrable on $(0, \infty)$.

Remark 4.7. The hypothesis that $\alpha \geq 0$ is given for convenience, as we only need the result in that case; however, the lemma is true and proved in the same way also for negative $\alpha$ with the additional requirement that $1+\alpha \geq k \geq \beta$, so that $\left\{y^{\alpha} g\right\}$ makes sense according to Definition 1.9. 
Remark 4.8. The result works also when $a$ is a linear combination of terms of the form (1.3) with the same $\lambda$. In this case, if $\alpha_{i}, \beta_{i}$ are the exponents in the $i$-th term in the linear combination, the proposition is true taking $\beta:=\max _{i}\left\{\beta_{i}\right\}$ and $\alpha:=\min _{i}\left\{\alpha_{i}\right\}$.

Proof. It is enough to prove it for a coagulation coefficient of the form (1.3) with $\alpha \geq 0$, as then we can apply the result to each term of the linear combination. For such a coefficient, the representation Theorem 3.3 shows we can write $C(g, g)$ as

$$
C(g, g)=\left\{y^{\alpha} g\right\} *\left\{y^{\beta} g\right\} .
$$

As $y \mapsto y^{k} g(y)$ is locally integrable on $[0, \infty)$, we know that

- $y^{k-\alpha} y^{\alpha} g$ is locally integrable on $[0, \infty)$, and

- $y^{k-\beta} y^{\beta} g$ is locally integrable on $[0, \infty)$,

and hence from Lemma 4.3

- $D^{\alpha-k}\left\{y^{\alpha} g\right\}$ is locally integrable on $\mathbb{R}$, and

- $D^{\beta-k}\left\{y^{\beta} g\right\}$ is locally integrable on $\mathbb{R}$, so $D^{\alpha-k}\left\{y^{\beta} g\right\}$ is also (as $\beta \geq \alpha$; see Lemma 8.11).

In addition, both $D^{\nu}\left\{y^{\alpha} g\right\}$ and $D^{\nu}\left\{y^{\beta} g\right\}$ are locally integrable on $(0, \infty)$, as $\left\{y^{\alpha} g\right\},\left\{y^{\beta} g\right\}$ are equal to the functions $y^{\alpha} g, y^{\beta} g$, respectively, on that set, and then Theorem 8.10 applies there. Hence, we obtain our result as an application of Lemma 4.5 with $S:=\left\{y^{\alpha} g\right\}, T:=\left\{y^{\beta} g\right\}$ and $\mu:=\alpha-k$

Now we can finally prove Theorem 1.1:

Proof of Theorem 1.1. Take any $\lambda<k<1$, with $\lambda$ the homogeneity degree of $a$. Then, $y^{k} g$ is locally integrable on $[0, \infty)$ as recalled in Proposition 4.1 (actually, we know it is integrable). We will show the following: if, for some $\nu \geq 0, D^{\nu} g$ is locally integrable on $(0, \infty)$, then $D^{\nu+1+\alpha-k} g$ is also locally integrable on $(0, \infty)$. As $\nu+1+\alpha-k>\nu$, this implies that $g$ is infinitely differentiable by a bootstrap argument starting with $\nu=0(g$ is locally integrable by definition).

To show this, write the equation for a self-similar profile as

$$
2 g+y D^{1} g+(1-\lambda) C(g, g)=0 \quad \text { as distributions on }(0, \infty) .
$$

Assume that $D^{\nu} g$ is locally integrable on $(0, \infty)$. Then, by Proposition 4.6, $D^{\nu+\alpha-k} C(g, g)$ is locally integrable on $(0, \infty)$, and hence eq. (4.11) shows that $D^{\nu+\alpha-k}\left(y D^{1} g\right)$ is locally integrable on $(0, \infty)$. By Theorem 8.10, the same is true of $D^{\nu+\alpha-k} D^{1} g=D^{\nu+\alpha-k+1} g$, which proves our claim. Hence, $g$ is infinitely differentiable. 


\section{Rewriting the self-similar equation}

One of the techniques that we use in order to study the behavior of scaling profiles near $y=0$ and the uniqueness of self-similar solutions is a way of rewriting equation (2.3) in which we "solve" the differential part of the equation as far as possible. We introduce this method next:

Lemma 5.1. (Solution of an o.d.e.). Let $g$ be an absolutely continuous function, and let $h, \mu$ be locally integrable functions, all of them defined on $(0, \infty)$. If the following equation holds

$$
\mu(y) g(y)+y g^{\prime}(y)=h(y) \quad \text { for almost all } y>0,
$$

then $g$ is given by

$$
g(y)=K(y) e^{-\Lambda(y)} \quad \text { for all } y>0,
$$

where $\Lambda$ and $K$ are absolutely continuous functions which satisfy that

$$
\begin{gathered}
\Lambda^{\prime}(y)=\frac{\mu(y)}{y} \quad \text { for almost all } y>0, \\
K^{\prime}(y)=\frac{1}{y} e^{\Lambda(y)} h(y) \quad \text { for almost all } y>0 .
\end{gathered}
$$

Proof. We remark that one may find the expression of $g$ by the method of variation of constants. To prove the result, a direct check shows that if we take functions $\Lambda, K$ satisfying (5.2), (5.3) and define

$$
\tilde{g}(y):=K(y) e^{-\Lambda(y)},
$$

then equation (5.1) holds with $\tilde{g}$ instead of $g$. We may add a constant to $K$ so that $g(1)=\tilde{g}(1)$, for instance. Now, if we regard (5.1) as an ordinary differential equation for $g$, then both $g$ and $\tilde{g}$ are solutions of it in the sense of Carathéodory, and then general uniqueness theorems (see, e.g., [8]) prove that $g=\tilde{g}$.

Lemma 5.2. (Primitive of $C$ ). Assume that the coagulation coefficient a is of the form (1.3) with $\alpha=0$, and take a function $g \in L_{1}^{1} \cap L_{\lambda}^{1}$ (this is, with finite mass and finite moment of order $\lambda)$. Then the primitive of $C(g, g)$ can be written as

$$
D_{-1} C(g, g)=G *\left(y^{\lambda} g\right)-M_{\lambda}[g] G,
$$

where $G$ is the function given by

$$
G(y):=\int_{y}^{\infty} g(z) d z \quad \text { for } y>0, \quad G(y):=0 \quad \text { for } y \leq 0 .
$$

We remark that this equality is an equality of distributions on $\mathbb{R}$. 
Remark 5.3. This is not a new result: one may arrive at the same expression by taking a characteristic function of the interval $(y, \infty)$ as the function $\phi$ in (2.2). The main point of the lemma is that it is rigorously proved as an equality of distributions.

Proof. The representation of $C(g, g)$ in Theorem 3.3 gives

$$
C(g, g)=\{g\} *\left\{y^{\lambda} g\right\}=\{g\} *\left(y^{\lambda} g\right)-M_{\lambda}[g]\{g\},
$$

as $g$ has finite $\lambda$-moment. Hence, taking the primitive $D_{-1}$ (see eq. (8.11)) we have

$$
D_{-1} C(g, g)=\left(D_{-1}\{g\}\right) *\left(y^{\lambda} g\right)-M_{\lambda}[g] D_{-1}\{g\}=G *\left(y^{\lambda} g\right)-M_{\lambda}[g] G,
$$

where we have used the derivation rule for a convolution (cf. theorem 8.8) and the fact that, as distributions on $\mathbb{R}$,

$$
D_{-1}\{g\}=G \text {. }
$$

Lemma 5.4. Assume that the coagulation coefficient a is of the form (1.3) with $\alpha=0$. If $g$ is a self-similar solution of Smoluchowski's equation, it holds that

$$
G(y)=y^{1-\tau} K(y)
$$

for some absolutely continuous function $K$, where $G$ is given by (5.5), and where

$$
\begin{aligned}
\tau & :=2-(1-\lambda) M_{\lambda}[g] \\
K^{\prime}(y) & =-y^{\tau-2} h(y) \quad \text { for almost all } y>0, \\
h & :=(1-\lambda)\left(G *\left(y^{\lambda} g\right)\right),
\end{aligned}
$$

We note that for the convolution in (5.10) it is assumed that $g(y)$ is 0 for $y<0$.

Remark 5.5. Note that the quantity $\tau$ here is in agreement with that in $[17,7]$.

Proof. It is known that in the case $\alpha=0$ the moment of order $\lambda$ of a solution $g$ is finite $[4,7]$. Hence, using (5.4) we can rewrite equation (1.4) by taking its primitive:

$$
G-y g+(1-\lambda)\left(G *\left(y^{\lambda} g\right)-M_{\lambda}[g] G\right)=0,
$$


which holds for all $y>0$. Equivalently,

$$
\left(1-(1-\lambda) M_{\lambda}[g]\right) G-y g+(1-\lambda)\left(G *\left(y^{\lambda} g\right)\right)=0 .
$$

Rewrite this as

$$
(\tau-1) G-y g+h=0,
$$

where $\tau$ and $h$ are given by (5.8) and (5.10). Now, if we solve for $G$ in equation (5.13) by using Lemma 5.1 with the independent term $h$, we obtain (5.7) and (5.9) in our result.

Lemma 5.6. Assume that the coagulation coefficient a is of the form (1.3) with $\alpha<0$, and take a self-similar solution $g$ of Smoluchowski's equation. Then it holds that

$$
g(y)=K(y) e^{-\Lambda(y)}
$$

for some absolutely continuous function $K$ such that

$$
K^{\prime}(y)=\frac{1}{y} e^{\Lambda(y)} h(y) \quad \text { for almost all } y>0,
$$

with

$$
\begin{aligned}
h & :=-(1-\lambda)\left(y^{\alpha} g\right) *\left(y^{\beta} g\right), \\
\Lambda(y) & :=2 \log y-(1-\lambda)\left(\frac{M_{\beta}}{\alpha} y^{\alpha}+\frac{M_{\alpha}}{\beta} y^{\beta}\right)
\end{aligned}
$$

when $\beta>0$, and

$$
\Lambda(y):=\left(2-(1-\lambda) M_{\alpha}\right) \log y-(1-\lambda) \frac{M_{\beta}}{\alpha} y^{\alpha}
$$

when $\beta=0$.

Proof. Let $g$ be a self-similar profile for Smoluchowski's coagulation equation with such a kernel $a$. It is known [4] that it is infinitely differentiable and has finite moments of all orders, and hence satisfies equation (1.4) in a strong way:

$$
2 g+y \partial_{y} g+(1-\lambda) C(g, g)=0,
$$

or, separating the gain and loss parts of $C(g, g)$,

$$
2 g+y \partial_{y} g+(1-\lambda)\left(y^{\alpha} g\right) *\left(y^{\beta} g\right)-(1-\lambda) M_{\beta} y^{\alpha} g-(1-\lambda) M_{\alpha} y^{\beta} g=0
$$

where $M_{\alpha}$ and $M_{\beta}$ are the moments of order $\alpha$ and $\beta$, respectively, of $g$. 
Now, if we apply Lemma 5.1 to equation (5.19) with the independent term $h$ given by $(5.16)$ and $\mu:=2-(1-\lambda)\left(M_{\beta} y^{\alpha}+M_{\alpha} y^{\beta}\right)$, we obtain that

$$
g(y)=K(y) e^{-\Lambda(y)},
$$

for some absolutely continuous functions $K, \Lambda$ such that

$$
\begin{gathered}
\Lambda^{\prime}(y)=\frac{1}{y}\left(2-(1-\lambda)\left(M_{\beta} y^{\alpha}+M_{\alpha} y^{\beta}\right)\right) \quad \text { a.e. } y>0, \\
K^{\prime}(y)=\frac{1}{y} e^{\Lambda(y)} h(y) \quad \text { a.e. } y>0 .
\end{gathered}
$$

We may actually choose $\Lambda$ as a particular primitive, as then the integration constant for $K$ can be adjusted so that (5.20) is still true. Then, we can take $\Lambda$ as in (5.17) (or (5.18) when $\beta=0$ ), and the result is proved.

\section{Asymptotic behavior at $y=0$ for kernels with $\alpha<0$}

In this section we prove Theorem 1.2. Assume that the coagulation coefficient $a$ is of the form (1.3) with $\alpha<0$. Let $g$ be a self-similar profile for Smoluchowski's coagulation equation with such a kernel $a$. Then from Lemma 5.6 we know it holds that

$$
g(y)=K(y) e^{-\Lambda(y)},
$$

for some absolutely continuous functions $K$ such that (5.15)-(5.16) hold, and with $\Lambda$ given by (5.17) (or (5.18) when $\beta=0$ ).

We will prove that $K$ is bounded on $(0, R)$ for any $R>0$. Obviously, $K$ is bounded on any interval $(\delta, R)$ with $0<\delta<R$, as is clear from (6.1), so the point is in proving that $K$ is bounded on $(0, \delta)$ for some $\delta>0$.

Then, take $\epsilon, \delta>0$ and define

$$
N_{\epsilon}:=\sup _{y \in(0, \delta)} g(y) \Phi_{\epsilon}(y)
$$

where

$$
\Phi_{\epsilon}(y):= \begin{cases}e^{\Lambda(\epsilon)} & \text { if } 0<y<\epsilon \\ e^{\Lambda(y)} & \text { if } \epsilon \leq y .\end{cases}
$$

The dependence of $N_{\epsilon}$ on $\epsilon$ is explicitly noted because we want to take the limit $\epsilon \rightarrow 0$; of course, $N_{\epsilon}$ depends also on $\delta$, but we do not write the dependence explicitly, as our intention is to fix $\delta$ at some value, which has not been chosen yet. We can give a bound for $g$ in terms of $N_{\epsilon}$ :

$$
g(y) \leq N_{\epsilon} \Phi_{\epsilon}(y)^{-1} \quad \text { for } y \in(0, \delta) .
$$


On the other hand, from (5.15),

$$
K(y)=K(\delta)-(1-\lambda) \int_{y}^{\delta} \frac{1}{z} e^{\Lambda(z)} h(z) d z \quad \text { for } y>0 .
$$

Let us find a bound for $h$ using (6.3) and our knowledge that $g$ is a bounded function [4]:

$$
g(y) \leq K_{1} \quad \text { for } y>0 .
$$

We have

$$
h=-(1-\lambda)\left(y^{\alpha} g\right) *\left(y^{\beta} g\right) .
$$

To bound this for $y \in(0, \delta)$, take $\delta$ small enough so that $e^{-\Lambda(y)}$ is increasing on $(0, \delta)$ (and so is $\Phi_{\epsilon}(y)^{-1}$ ), and then

$$
\left(y^{\alpha} g\right) *\left(y^{\beta} g\right) \leq N_{\epsilon} K_{1} \Phi_{\epsilon}(y)^{-1}\left(y^{\alpha}\right) *\left(y^{\beta}\right)=N_{\epsilon} K_{2} \Phi_{\epsilon}(y)^{-1} y^{\lambda+1}
$$

for $y \in(0, \delta)$. Then, from (6.6),

$$
|h(y)| \leq N_{\epsilon} K_{3} \Phi_{\epsilon}(y)^{-1} y^{\lambda+1}
$$

and continuing from (6.4), taking into account that $e^{\Lambda(z)} \Phi_{\epsilon}(z)^{-1}$ is decreasing on $(0, \delta)$,

$$
\begin{aligned}
|K(y)| & \leq K(\delta)+(1-\lambda) N_{\epsilon} K_{3} \int_{y}^{\delta} z^{\lambda} e^{\Lambda(z)} \Phi_{\epsilon}(z)^{-1} d z \\
& \leq K(\delta)+N_{\epsilon} K_{4} e^{\Lambda(y)} \Phi_{\epsilon}(y)^{-1} \int_{y}^{\delta} z^{\lambda} d z \\
& \leq K(\delta)+N_{\epsilon} K_{5} e^{\Lambda(y)} \Phi_{\epsilon}(y)^{-1} \delta^{\lambda+1} \quad \text { for } y \in(0, \delta) .
\end{aligned}
$$

Hence, multiplying by $e^{-\Lambda(y)} \Phi_{\epsilon}(y)$ (which is always less than 1), taking into account that $g \Phi_{\epsilon}=K e^{-\Lambda} \Phi_{\epsilon}$, and taking the supremum over $(0, y)$,

$$
N_{\epsilon} \leq K(\delta)+N_{\epsilon} K_{5} \delta^{\lambda+1},
$$

As $\lambda+1>0$, taking $\delta$ small enough gives a bound for $N_{\epsilon}$ which is independent of $\epsilon$, and hence proves that $K$ is bounded on $(0, \delta)$. This in turn implies that it has a strictly positive limit at $y=0$, as it is a nonincreasing function, which can be seen from equations (5.15)-(5.16). 


\section{Partial uniqueness of scaling profiles}

Let us prove Theorems 1.6 and 1.7 on the partial uniqueness of scaling profiles. We will prove the following proposition, which already contains Theorem 1.7, and which will be seen to easily imply Theorem 1.6 (see Section 7.2):

Proposition 7.1. Assume that the coagulation coefficient $a$ is of the form (1.3) with $\alpha \leq 0$. Assume that $g^{1}$ and $g^{2}$ are two self-similar profiles for Smoluchowski's equation, and also that

1. in the case $\alpha=0, M_{\lambda}\left[g_{1}\right]=M_{\lambda}\left[g_{2}\right]$, and

$$
\lim _{y \rightarrow 0} y^{\tau-1} G^{1}(y)=\lim _{y \rightarrow 0} y^{\tau-1} G^{2}(y),
$$

where $\tau$ is given by (5.8), and $G^{1}, G^{2}$ are the primitives of $g^{1}, g^{2}$ based at $+\infty$, defined as in (5.5).

2. in the case $\alpha<0, M_{\alpha}\left[g_{1}\right]=M_{\alpha}\left[g_{2}\right], M_{\beta}\left[g_{1}\right]=M_{\beta}\left[g_{2}\right]$, and

$$
\lim _{y \rightarrow 0} g^{1}(y) e^{\Lambda(y)}=\lim _{y \rightarrow 0} g^{2}(y) e^{\Lambda(y)},
$$

where $\Lambda$ is given by (5.17).

Then, $g^{1}=g^{2}$.

We prove this proposition in two parts: in the next section we prove that the result holds near $y=0$, and in Section 7.2 we prove that the result is global.

\subsection{Local result}

We will start by proving Proposition 7.1 locally near $y=0$; this is, we will show that under the same hypotheses there exists $\delta>0$ such that $g^{1}(y)=g^{2}(y)$ for $0<y<\delta$.

\subsubsection{Proof for $\alpha=0$}

Step 1: Rewriting the equation for a difference of profiles. Assume that the coagulation coefficient $a$ is given by (1.3) with $\alpha=0$ (so $\beta=\lambda$ ). If $g$ is a self-similar profile, then (5.7)-(5.10) from lemma 5.4 hold, so

$$
K(y)=K_{0}-\int_{0}^{y} z^{\tau-2} h(z) d z
$$

with $K_{0}$ given by

$$
K_{0}:=\lim _{y \rightarrow 0} K(y)=\lim _{y \rightarrow 0} y^{\tau-1} G(y),
$$


which is known to exist and be strictly positive [7]. Note that here we have integrated equation (5.9) between 0 and $y$, which can be done once we know $K$ has a limit at $y=0$.

Gathering the above, we have

$$
y^{\tau-1} G(y)=K_{0}-(1-\lambda) \int_{0}^{y} z^{\tau-2}\left(G *\left(y^{\lambda} g\right)\right)(z) d z,
$$

which is a remarkable equation in the sense that it is local near 0 , and the term to the right is more regular near 0 than that to the left, as will be precised later. We also remark that in (7.3) the parameters $\tau$ and $K_{0}$ depend on $g$.

Now, let us obtain the corresponding equation for the difference of two self-similar profiles. Let $g_{1}, g_{2}$ be two solutions of self-similar profiles, and assume that they satisfy the conditions in Proposition 7.1. Then, both $g_{1}$ and $g_{2}$ satisfy (7.3) with the same $\tau$ and $K_{0}$, and we can take the difference to get

$$
\begin{aligned}
y^{\tau-1} \Delta G(y)= & -\frac{1-\lambda}{2} \int_{0}^{y} z^{\tau-2}\left((\Delta G) *\left(y^{\lambda} g_{1}\right)\right)(z) d z \\
& -\frac{1-\lambda}{2} \int_{0}^{y} z^{\tau-2}\left(G_{2} *\left(y^{\lambda} \Delta g\right)\right)(z) d z
\end{aligned}
$$

where

$$
\Delta g:=g_{1}-g_{2}, \quad \Delta G:=G_{1}-G_{2} .
$$

Now, to obtain local uniqueness near 0 , take $\epsilon>0$ and write

$$
N \equiv N(\epsilon):=\sup _{y \in(0, \epsilon)} y^{\tau-1}|\Delta G(y)|
$$

Observe that this quantity is known to be bounded thanks to [7]. Let us prove from equation (7.4) that, if we take $\epsilon$ small enough, then $N$ must be 0 .

Step 2: Estimate for the first term. Constants independent of $\epsilon$ will be denoted by $K_{1}, K_{2}$. . We will use the following bound, which holds [7] for all solutions $g$ of (1.4) (and in particular for $g_{1}$ and $g_{2}$ ):

$$
g(y) \leq K_{1} y^{-\tau} \quad \text { for } y>0
$$

for some constant $K_{1}>0$, which implies that

$$
G(y) \leq K_{2} y^{1-\tau} \quad \text { for } y>0,
$$


for some other constant $K_{2}$. Then, from (7.6),

$$
\begin{aligned}
\left|(\Delta G) *\left(y^{\lambda} g_{1}\right)(z)\right| & \leq \int_{0}^{z}|\Delta G(x)|(x-z)^{\lambda}\left|g_{1}(x-z)\right| d x \\
& \leq K_{1} N \int_{0}^{z} x^{1-\tau}(x-z)^{\lambda-\tau} d x \leq K_{3} N y^{2+\lambda-2 \tau}
\end{aligned}
$$

Hence, the first term on the right hand side of (7.4) can be estimated by

$$
\left|\int_{0}^{y} z^{\tau-2}\left((\Delta G) *\left(y^{\lambda} g_{1}\right)\right)(z) d z\right| \leq N K_{3} \int_{0}^{y} z^{\lambda-\tau} d z=N K_{4} y^{1+\lambda-\tau}
$$

Step 3: Estimate for the second term. For the second term in (7.4) we need to make $\Delta G$ appear instead of $\Delta g$. We use integration by parts to write:

$$
\begin{aligned}
& \int_{0}^{y} z^{\tau-2}\left(G_{2} *\right.\left.\left(y^{\lambda} \Delta g\right)\right)(z) d z= \\
&=y^{\tau-2} \int_{0}^{y}\left(G_{2} *\left(y^{\lambda} \Delta g\right)\right)(z) d z \\
& \quad+(2-\tau) \int_{0}^{y} z^{\tau-3} \int_{0}^{z}\left(G_{2} *\left(y^{\lambda} \Delta g\right)\right)(x) d x d z
\end{aligned}
$$

Here, the boundary term at $y=0$ in the integration by parts vanishes, which is a consequence of the bound below in eq. (7.15), which we will show next for the term $\int_{0}^{y} G_{2} *\left(y^{\lambda} \Delta g\right)$ appearing above. Write:

$$
\begin{aligned}
\int_{0}^{y}\left(G_{2} *\left(y^{\lambda} \Delta g\right)\right)(z) d z & =D^{-1}\left(G_{2} *\left(y^{\lambda} \Delta g\right)\right)(z) d z \\
& =\left(G_{2} * D^{-1}\left(y^{\lambda} \Delta g\right)\right)(y) .
\end{aligned}
$$

Also,

$$
D^{-1}\left(y^{\lambda} \Delta g\right)(y)=-y^{\lambda} \Delta G(y)+\lambda \int_{0}^{y} z^{\lambda-1} \Delta G(z) d z
$$

So

$$
\begin{aligned}
\left|D^{-1}\left(y^{\lambda} \Delta g\right)(y)\right| & \leq\left|y^{\lambda} \Delta G(y)\right|+\lambda \int_{0}^{y} z^{\lambda-1}|\Delta G(z)| d z \\
& \leq N y^{1-\tau+\lambda}+N \lambda \int_{0}^{y} z^{\lambda-\tau} d z \leq K_{5} N y^{1+\lambda-\tau} .
\end{aligned}
$$


Hence, from (7.11),

$$
\begin{aligned}
& \left|\int_{0}^{y}\left(G_{2} *\left(y^{\lambda} \Delta g\right)\right)(z) d z\right| \leq\left|\left(G_{2} * D^{-1}\left(y^{\lambda} \Delta g\right)\right)(y)\right| \\
& \leq N K_{2} K_{5} \int_{0}^{y} z^{1-\tau}(y-z)^{1+\lambda-\tau} d z \leq N K_{6} y^{3+\lambda-2 \tau} .
\end{aligned}
$$

And finally, gathering (7.10) and (7.14),

$$
\begin{aligned}
\mid \int_{0}^{y} z^{\tau-2} & \left(G_{2} *\left(y^{\lambda} \Delta g\right)\right)(z) d z \mid \leq \\
& \leq N K_{6} y^{\tau-2} y^{3+\lambda-2 \tau}+N K_{6}|2-\tau| \int_{0}^{y} z^{\tau-3} z^{3+\lambda-2 \tau} d z \\
& \leq N K_{7} y^{1+\lambda-\tau} .
\end{aligned}
$$

Step 4: Final estimate Now, from equation (7.4), taking the supremum on $(0, \epsilon)$ and using (7.9) and (7.15) one has

$$
N(\epsilon) \leq N(\epsilon) K_{8} \epsilon^{1+\lambda-\tau} .
$$

Note that $1+\lambda-\tau>0$ [7], which is crucial for this argument, and is a particular property of the coagulation kernel we are using. Hence, for $\epsilon>0$ small enough, we have that

$$
N \equiv N(\epsilon)=0
$$

and hence that

$$
g_{1}(y)=g_{2}(y) \quad \text { for } 0<y<\epsilon,
$$

which proves the equality in Proposition 7.1 locally near $y=0$ in the case $\alpha=0$.

\subsubsection{Proof for $\alpha<0$}

Assume again that $a$ is of the form (1.3), now with $\alpha<0$. Take two selfsimilar solutions $g_{1}, g_{2}$ in the conditions of Proposition 7.1. Then Lemma 5.6 applies to both $g_{1}$ and $g_{2}$ with the same $\Lambda$, and following the same reasoning as in the $\alpha=0$ case we have

$$
\Delta g(y)=\Delta K(y) e^{-\Lambda(y)},
$$

with

$$
\begin{aligned}
(\Delta K)^{\prime}(y) & =\frac{1}{y} e^{\Lambda(y)} \Delta h(y) \quad \text { for almost all } y>0, \\
\Delta h & =-(1-\lambda)\left(y^{\alpha} \Delta g\right) *\left(y^{\beta} g_{2}\right)-(1-\lambda)\left(y^{\alpha} g_{2}\right) *\left(y^{\beta} \Delta g\right) .
\end{aligned}
$$

Here we denote $\Delta A:=A_{1}-A_{2}$ for any function $A$, and $K_{1}, h_{1}, K_{2}, h_{2}$ are the functions $K, h$ associated to $g_{1}, g_{2}$ as in Lemma 5.6. 
Then, from the equality of the limits in (1.9) we see that $\Delta K(y) \rightarrow 0$ as $y \rightarrow 0$, so integrating (7.18),

$$
\Delta K(y)=\int_{0}^{y} \frac{1}{z} e^{\Lambda(z)} \Delta h(z) d z .
$$

Let us find a bound for $\Delta h$ using $\Delta K$. Take $\epsilon>0$ and call

$$
N:=\sup _{y \in(0, \epsilon)}|\Delta K(y)| .
$$

Then, for the first term in (7.19),

$$
\begin{aligned}
\left|\left(y^{\alpha} \Delta g\right) *\left(y^{\beta} g_{2}\right)\right| & \leq N\left(y^{\alpha} e^{-\Lambda(y)}\right) *\left(y^{\beta} g_{2}\right) \\
& \leq N K_{0} e^{-\Lambda(y)}\left(y^{\beta}\right) *\left(y^{\alpha}\right) \leq N K_{1} e^{-\Lambda(y)} y^{\lambda+1},
\end{aligned}
$$

where we have also used that $g$ is bounded on $(0, \epsilon)$; of course, we have much stronger information on its behavior at $y=0$, which has been used through the fact that $\Delta K$ is bounded and has a limit at $y=0$.

For the second term in (7.19) a similar calculation shows that

$$
\left|\left(y^{\alpha} g_{2}\right) *\left(y^{\beta} \Delta g\right)\right| \leq N K_{2} y^{\lambda+1} e^{-\Lambda(y)} .
$$

Putting together (7.22) and (7.23) we obtain a bound for $\Delta h$ :

$$
|\Delta h(y)| \leq N K_{3} y^{\lambda+1} e^{-\Lambda(y)},
$$

and continuing from (7.20),

$$
|\Delta K(y)| \leq N K_{3} \int_{0}^{y} z^{\lambda} d z=N K_{4} y^{\lambda+1} .
$$

Finally, taking the supremum on $(0, \epsilon)$,

$$
N \leq N K_{4} \epsilon^{\lambda+1},
$$

and then (as $\lambda+1>0$ ) choosing $\epsilon$ small enough proves that $N=0$. Hence,

$$
g_{1}(y)=g_{2}(y) \quad \text { for } y \in(0, \epsilon) .
$$

\subsection{Global result}

We now extend the local result in the previous section in order to finish the proof of Proposition 7.1. After this it will also be easy to prove Theorem 1.6.

End of Proof of Proposition 7.1. Take two self-similar profiles $g_{1}$ and $g_{2}$ satisfying the hypotheses of the theorem. The results in previous sections show that $G_{1}=G_{2}$ on some interval $\left(0, y_{0}\right]$, for some $y_{0}>0$. Following a 
strategy usual in uniqueness theorems for ordinary differential equations, we will show that whenever $G_{1}=G_{2}$ on an interval $\left(0, y_{0}\right]$, there is a $\delta>0$ such that $G_{1}=G_{2}$ on $\left(0, y_{0}+\delta\right)$. Together with our local uniqueness result, a well-known argument then shows that $G_{1}=G_{2}$, and so $g_{1}=g_{2}$, on $(0, \infty)$.

So, assume that $G_{1}=G_{2}$ on $\left(0, y_{0}\right]$ for some $y_{0}>0$. We take $\delta>0$ (to be fixed later), and intend to prove that $G_{1}=G_{2}$ on $\left(0, y_{0}+\delta\right)$.

Proof for $\boldsymbol{\alpha}<\mathbf{0}$. We follow the notation in Section 7.1.2 and call

$$
N:=\sup _{y \in\left(0, y_{0}+\delta\right)}|\Delta K(y)|,
$$

in analogy with (7.21). From (7.20), and taking into account that $\Delta h=0$ on $\left(0, y_{0}\right)$,

$$
N=\|\Delta K(y)\|_{L^{\infty}\left(0, y_{0}+\delta\right)} \leq \int_{y_{0}}^{y_{0}+\delta} \frac{1}{z} e^{\Lambda(z)}|\Delta h(z)| d z .
$$

Now, using the bound (7.24),

$$
N \leq N K_{3} \int_{y_{0}}^{y_{0}+\delta} z^{\lambda} d z \leq N K_{3} \delta\left(y_{0}+1\right)^{\lambda},
$$

which implies that $N=0$ provided we take $\delta$ small enough.

Proof for $\boldsymbol{\alpha}=\mathbf{0}$. We now follow the notation in Section 7.1.1, and call

$$
N:=\sup _{y \in\left(0, y_{0}+\delta\right)} y^{\tau-1}|\Delta G(y)| .
$$

as in (7.5). We have eq. (7.4):

$$
\begin{aligned}
y^{\tau-1} \Delta G(y)= & -\frac{1-\lambda}{2} \int_{0}^{y} z^{\tau-2}\left((\Delta G) *\left(y^{\lambda} g_{1}\right)\right)(z) d z \\
& -\frac{1-\lambda}{2} \int_{0}^{y} z^{\tau-2}\left(G_{2} *\left(y^{\lambda} \Delta g\right)\right)(z) d z,
\end{aligned}
$$

and we can follow the reasoning leading to the estimate (7.9) of the first term, and take into account $G_{1}=G_{2}$ on $\left(0, y_{0}\right]$, to obtain

$$
\left|\int_{0}^{y} z^{\tau-2}\left((\Delta G) *\left(y^{\lambda} g_{1}\right)\right)(z) d z\right| \leq N K_{3} \int_{y_{0}}^{y_{0}+\delta} z^{\lambda-\tau} d z \leq N \delta K_{4} y_{0}^{\lambda-\tau}
$$

for $y \in\left(y_{0}, y_{0}+\delta\right)$. 
For the second term, we repeat the reasoning leading to (7.14) to obtain instead, for $y \in\left(y_{0}, y_{0}+\delta\right)$

$$
\begin{aligned}
\mid \int_{0}^{y}\left(G_{2} *\left(y^{\lambda} \Delta g\right)\right) & (z) d z|\leq|\left(G_{2} * D^{-1}\left(y^{\lambda} \Delta g\right)\right)(y) \mid \\
\leq & N K_{2} K_{5} \int_{y_{0}}^{y} z^{1+\lambda-\tau}(y-z)^{1-\tau} d z \\
& \leq N K_{2} K_{5}\left(y_{0}+1\right)^{1+\lambda-\tau} \int_{y_{0}}^{y}(y-z)^{1-\tau} d z \leq N K_{9} \delta^{2-\tau},
\end{aligned}
$$

for some number $K_{9}$ independent of $\delta$. With this we obtain the corresponding to (7.15):

$$
\left|\int_{0}^{y} z^{\tau-2}\left(G_{2} *\left(y^{\lambda} \Delta g\right)\right)(z) d z\right| \leq N K_{10} \delta^{2-\tau}
$$

for $y \in\left(y_{0}, y_{0}+\delta\right)$ and some number $K_{10}$. Using (7.30) and (7.29) in (7.28), and taking the supremum on $\left(0, y_{0}+\delta\right)$,

$$
N \leq \frac{1-\lambda}{2}\left(N \delta K_{4} y_{0}^{\lambda-\tau}+N \delta^{2-\tau} K_{10}\right),
$$

which shows $N=0$ once we take $\delta$ small enough.

Let us finally prove Theorem 1.6:

Proof of Theorem 1.6. Assume that $a$ is of the form (1.3) with $\alpha=0$, and take two solutions $g_{1}, g_{2}$ of equation (1.4) in the conditions of Theorem 1.6, this is,

$$
\begin{aligned}
\int_{0}^{\infty} y g_{1}(y) d y & =\int_{0}^{\infty} y g_{2}(y) d y \\
\int_{0}^{\infty} y^{\lambda} g_{1}(y) d y & =\int_{0}^{\infty} y^{\lambda} g_{2}(y) d y .
\end{aligned}
$$

Then, for any $\mu>0$, the function

$$
\tilde{g}_{1}(y):=\mu^{1+\lambda} g_{1}(\mu y) \quad \text { for } y>0
$$

is another solution of equation (1.4), which is a simple consequence of the homogeneity of the coagulation coefficient $a$ (see, for example, [7]). We also check easily that

$$
\begin{aligned}
\int_{0}^{\infty} y^{\lambda} \tilde{g}_{1}(y) d y & =\int_{0}^{\infty} y^{\lambda} g_{1}(y) d y \\
\int_{0}^{\infty} y \tilde{g}_{1}(y) d y & =\mu^{\lambda-1} \int_{0}^{\infty} y g_{1}(y) d y \\
\lim _{y \rightarrow 0} y^{\tau-1} \int_{y}^{\infty} \tilde{g}_{1}(z) d z & =\mu^{1+\lambda-\tau} \lim _{y \rightarrow 0} y^{\tau-1} \int_{y}^{\infty} g_{1}(z) d z
\end{aligned}
$$


where $\tau:=2-(1-\lambda) M_{\lambda}\left[g_{1}\right]$. Hence, the moment of order $\lambda$ of $\tilde{g}_{1}$ is the same no matter which $\mu$ we take, so we can choose $\mu>0$ in such a way that

$$
\begin{aligned}
\int_{0}^{\infty} y^{\lambda} \tilde{g}_{1}(y) d y & =\int_{0}^{\infty} y^{\lambda} g_{2}(y) d y \\
\lim _{y \rightarrow 0} y^{\tau-1} \int_{y}^{\infty} \tilde{g}_{1}(z) d z & =\lim _{y \rightarrow 0} y^{\tau-1} \int_{y}^{\infty} g_{2}(z) d z .
\end{aligned}
$$

Hence, with this value of $\mu$, by Proposition 7.1 we have that $\tilde{g}_{1}=g_{2}$, and in particular their masses are equal; as the masses of $g_{1}$ and $g_{2}$ are also equal, from (7.32) we deduce that in fact $\mu$ must be equal to 1 , so $g_{1}=\tilde{g}_{1}=g_{2}$, which shows the result.

\section{Appendix: fractional derivatives}

The extension of the concept of integration and differentiation to include derivatives and integrals of noninteger order is a well established theory [16, $15,14]$. Here we give a brief but self-contained introduction to it and state without proof the main standard results. Some particular properties needed in the rest of this paper and which are not commonly encountered will be given with complete proofs below.

For our purposes, the simplest and most general definition of fractional derivatives is given in the context of distributions, and can be found in the book by Schwartz [16, VI.5]. The reader can check that our definitions are the same as those given there, even if the presentation is somewhat different. Other expositions are found in $[15,14]$, and we refer to those sources for the proof of the main results on fractional differentiation given below.

In the following we will use the space $\mathcal{C}_{R}^{\infty}$ consisting of all infinitely differentiable functions $f: \mathbb{R} \rightarrow \mathbb{R}$ which have support bounded below; this is, those $f$ which have support contained in $[a,+\infty)$ for some $a \in \mathbb{R}$. The notation $\mathcal{C}_{R}^{\infty}$ is intended to suggest that the important part of a function $f$ is to the right, if one represents the real line as usual. ${ }^{1}$ Analogously, we define $\mathcal{C}_{L}^{\infty}$ as the set of all infinitely differentiable functions $f: \mathbb{R} \rightarrow \mathbb{R}$ whose support is bounded above.

We will first define fractional derivatives for smooth functions, and then extend the concept to distributions with a common duality method.

\footnotetext{
${ }^{1}$ We prefer to write $\mathcal{C}_{R}^{\infty}$ instead of $\mathcal{D}_{+}$, used in Schwartz's book, as in the literature related to Smoluchowski's equation the subscript ' + ' is frequently used to denote that functions in the involved space are nonnegative, which could cause confusion here, where we want to stress a property of their support.
} 


\subsection{Fractional derivatives of smooth functions}

Definition 8.1. (Left fractional derivatives). For $f \in \mathcal{C}_{R}^{\infty}$ and real $k>0$, we define the left integral or order $k$ of $f$ as

$$
D^{-k} f(y):=\frac{1}{\Gamma(k)} \int_{-\infty}^{y} f(z)(y-z)^{k-1} d z \quad \text { for } y \in \mathbb{R},
$$

where $\Gamma$ is the Gamma function. For $k=0$ we just write $D^{k} f=f$. For real $k \geq 0$ we write $k$ as $k=n-s$, with $n>0$ an integer and $0 \leq s<1$, and define the left derivative of order $k$ of $f$ to be

$$
D^{k} f:=\frac{d^{n}}{d y^{n}}\left(D^{-s} f\right)
$$

The above is a usual definition of fractional integrals and derivatives [15], sometimes called the Riemann-Lebesgue definition. Names given to the above also differ slightly from place to place: we may refer to $D^{k} f$ as the left derivative of order $k$ of $f$, or just the $k$-th derivative of $f$, for any real $k$ (even for $k<0$ ), thus emphasizing that all $D^{k}$ are part of a family of operators with common properties.

There is a completely analogous concept of right derivative where integrals are taken from $+\infty$ :

Definition 8.2 (Right fractional derivatives). For $f \in \mathcal{C}_{L}^{\infty}$ and real $k>0$, we define the right integral or order $k$ of $f$ as

$$
D_{-k} f(y):=\frac{1}{\Gamma(k)} \int_{y}^{\infty} f(z)(z-y)^{k-1} d z \quad \text { for } y \in \mathbb{R},
$$

where $\Gamma$ is the Gamma function. For $k=0$ we just write $D_{k} f=f$. For real $k \geq 0$ we write $k$ as $k=n-s$, with $n>0$ an integer and $0 \leq s<1$, and define the left derivative of order $k$ of $f$ to be

$$
D_{k} f:=(-1)^{n} \frac{d^{n}}{d y^{n}}\left(D_{-s} f\right) \text {. }
$$

Some easy consequences are the following:

1. For $k \in \mathbb{R}$ and $f \in \mathcal{C}_{R}^{\infty}, D^{k} f$ is again on $\mathcal{C}_{R}^{\infty}$, and the analogous result holds for $D_{k}$ and $\mathcal{C}_{L}^{\infty}$.

2. For integer $k \geq 0, D^{k}$ is just the usual $k$-th derivative of $f$, while $D^{-k}$ is the $k$-fold iteration of the primitive based at $-\infty$; in particular, $D^{-1} f$ is the only primitive of $f$ which is 0 at $-\infty$. Analogously, $D_{k}$ is the usual $k$-th derivative of $f$, times $(-1)^{k}$ (see next for a natural reason for this definition), while $D_{-k}$ is the $k$-fold iteration of the primitive based at $+\infty$. 
3. Right derivatives are the concept symmetric to that of left derivatives under the reflection of $\mathbb{R}$ : if we define the reflection of a function $f: \mathbb{R} \rightarrow \mathbb{R}$ to be the function $\mathcal{R} f: \mathbb{R} \rightarrow \mathbb{R}$ given by $\mathcal{R} f(y):=f(-y)$, then

$$
D_{k}(f):=\mathcal{R}\left(D^{k}(\mathcal{R} f)\right) \quad \text { for } k \in \mathbb{R}, f \in \mathcal{C}_{L}^{\infty} .
$$

Alternatively, one can take this as a definition of right derivatives from the perhaps more natural concept of left derivatives. Note that the alternating sign in equation (8.4) is unavoidable if we want to conserve this symmetry property.

Remarkably, the following composition result holds:

Theorem 8.3. For real $k, j$,

$$
\begin{array}{ll}
D^{j}\left(D^{k} f\right)=D^{j+k} f & \text { for any } f \in \mathcal{C}_{R}^{\infty}, \\
D_{j}\left(D_{k} f\right)=D_{j+k} f & \text { for any } f \in \mathcal{C}_{L}^{\infty} .
\end{array}
$$

A proof follows from elementary analysis arguments. For this result to hold it is essential that our Definitions 8.1 and 8.2 above have picked specific primitives (those which are 0 at $-\infty$ or $+\infty$, respectively) out of all the possible primitives of a function $f$. Said in another way, the spaces $C_{R}^{\infty}$, $C_{L}^{\infty}$ in which we are working only contain one of all the possible primitives of a given function $f$, and thus the above composition rule can hold.

Also, it is easy to see that $D_{k}$ is the dual of $D^{k}$ in the following sense:

Lemma 8.4. For $f \in \mathcal{C}_{R}^{\infty}$ and $g \in \mathcal{C}_{L}^{\infty}$ it holds that

$$
\int_{-\infty}^{+\infty} D^{k} f(y) g(y) d y=\int_{-\infty}^{+\infty} f(y) D_{k} g(y) d y
$$

This suggests the definition for distributions given below.

\subsection{Fractional derivatives of distributions}

Consider the set $\mathcal{D}_{L}^{\prime}$ of distributions on $\mathbb{R}$ which have compact support bounded below. One can show that $\mathcal{D}_{L}^{\prime}$ is the dual of $\mathcal{C}_{L}^{\infty}$ when the latter is equipped with a natural topology (which extends that of $\mathcal{C}_{0}^{\infty}$ ) [16, VI.5]. So, $\mathcal{D}_{L}^{\prime}$ should be thought of as $\left(\mathcal{C}_{L}^{\infty}\right)^{\prime}$, which is useful for remembering that distributions in this space have support contained in $(a, \infty)$ for some $a \in \mathbb{R}$. For these distributions, one can define $\langle T, \psi\rangle$ for any $\psi \in \mathcal{C}_{L}^{\infty}$ as

$$
\langle T, \psi\rangle:=\langle T, \tilde{\psi}\rangle
$$

for any $\tilde{\psi} \in \mathcal{C}_{0}^{\infty}(\mathbb{R})$ which coincides with $\psi$ on the support of $T$. Of course, this definition does not depend on the particular extension chosen. We define $\mathcal{D}_{L}^{\prime}$ analogously, and also the pairing $\langle T, \phi\rangle$ for any $T \in \mathcal{D}_{L}^{\prime}, \phi \in \mathcal{C}_{R}^{\infty}$. 
Definition 8.5. (Fractional derivatives of distributions). Take $k \in \mathbb{R}$. For a distribution $T \in \mathcal{D}_{L}^{\prime}$ we define the distribution $D^{k} T$ as

$$
\left\langle D^{k} T, \psi\right\rangle:=\left\langle T, D_{k} \psi\right\rangle \quad \text { for } \psi \in \mathcal{C}_{L}^{\infty}(\mathbb{R}) .
$$

Analogously, for a distribution $T \in \mathcal{D}_{R}^{\prime}$ we define the distribution $D_{k} T$ as

$$
\left\langle D_{k} T, \psi\right\rangle:=\left\langle T, D^{k} \phi\right\rangle \quad \text { for } \phi \in \mathcal{C}_{L}^{\infty}(\mathbb{R}) .
$$

Here, $D^{k} \phi$ and $D_{k} \psi$ are the right and left fractional derivatives, respectively, defined in Section 8.1. Note that the duality products here are well defined as indicated in (8.9). Also, this agrees with Definitions 8.1 and 8.2 when $T$ is a function in $\mathcal{C}_{R}^{\infty}$ (or $\left.\mathcal{C}_{L}^{\infty}\right)$, as can be seen from (8.8).

Then, the $D^{k}$ are linear operators for which the composition rule (8.6) still holds: for any $j, k \in \mathbb{R}$,

$$
\begin{array}{ll}
D^{j}\left(D^{k} T\right)=D^{j+k} T & \text { for any } T \in \mathcal{D}_{R}^{\prime} \\
D_{j}\left(D_{k} T\right)=D_{j+k} T & \text { for any } T \in \mathcal{D}_{L}^{\prime} .
\end{array}
$$

The convolution of two distributions in $\mathcal{D}_{R}^{\prime}$ (or two distributions in $\mathcal{D}_{L}^{\prime}$ ) is well defined [16, VI.5], as we show below:

Definition 8.6. (Convolution of a distribution and a smooth function). Given $T \in \mathcal{D}_{R}^{\prime}$ and $\phi \in \mathcal{C}_{L}^{\infty}$ we define $T * \phi$ as the distribution in $\mathcal{D}_{R}^{\prime}$ given by

$$
\langle T * \phi, \psi\rangle:=\langle T,(\mathcal{R} \phi) * \psi\rangle \quad \text { for } \psi \in \mathcal{C}_{R}^{\infty} .
$$

Note that $(\mathcal{R} \phi) * \psi \in \mathcal{C}_{R}^{\infty}$. For $T \in \mathcal{D}_{L}^{\prime}$ and $\psi \in \mathcal{C}_{R}^{\infty}$ the convolution $T * \psi$ is defined analogously.

The convolution with a function in $\mathcal{C}_{R}^{\infty}\left(\right.$ or $\left.\mathcal{C}_{L}^{\infty}\right)$ is regularizing, as it happens in the more familiar case of convolution with a $C^{\infty}$ function with bounded support: if $T \in \mathcal{D}_{L}^{\prime}$ and $\psi \in \mathcal{C}_{R}^{\infty}$, then one can prove that $T * \psi$ is equal to a function in $\mathcal{C}_{R}^{\infty}$, given by

$$
T * \psi(y)=\left\langle T, \tau_{y} \psi\right\rangle \quad \text { for } y \in \mathbb{R},
$$

where $\left(\tau_{y} \psi\right)(x):=\psi(x-y)$ is the translation of $\psi$ by $y$. Similarly the convolution $T * \phi$ for $T \in \mathcal{D}_{R}^{\prime}$ and $\phi \in \mathcal{C}_{L}^{\infty}$ is a function in $\mathcal{C}_{L}^{\infty}$.

Definition 8.7. (Convolution of two distributions). Given $T, S \in \mathcal{D}_{R}^{\prime}$, we define $T * S$ as the distribution in $\mathcal{D}_{R}^{\prime}$ given by

$$
\langle T * S, \psi\rangle:=\langle T,(\mathcal{R} S) * \psi\rangle \quad \text { for } \psi \in \mathcal{C}_{R}^{\infty} .
$$

(As remarked before $(8.15),(\mathcal{R} S) * \psi \in \mathcal{C}_{R}^{\infty}$.) The convolution $T * S$ for $T, S \in \mathcal{D}_{L}^{\prime}$ is defined analogously. 
The following well-known result on the derivation of a convolution holds in complete generality with these definitions:

Theorem 8.8. For any $T, S \in \mathcal{D}_{L}^{\prime}$ and any $k \in \mathbb{R}$,

$$
D^{k}(T * S)=\left(D^{k} T\right) * S .
$$

Although fractional derivation and integration operators of non-integer order are not local, their regularity properties nevertheless depend only on local properties of the function they act on, as can be easily proved by observing that $D^{k} f$ can be written as a convolution of $f$ with a distribution which is $\mathcal{C}^{\infty}$ away from 0 [16, VI.5, p. 174]. A manifestation of this is the following result:

Theorem 8.9. Let $T$ be a distribution on $\mathbb{R}$ with compact support to the left (this is, $T \in \mathcal{D}_{L}^{\prime}$ ), and such that $T$ is $\mathcal{C}^{\infty}(U)$, for $U \subseteq \mathbb{R}$ a given open set. Then, $D^{k} T \in \mathcal{C}^{\infty}(U)$ for all $k \in \mathbb{R}$.

It is well known that multiplying a distribution by a $\mathcal{C}^{\infty}$ function preserves its local regularity, and this is still true when one measures this regularity in terms of the integrability of a given fractional derivative. As this result is not easily found in the literature and its proof is not obvious, we give it in Section 8.3 below:

Theorem 8.10. Let $T$ be a distribution on $\mathbb{R}$ with compact support to the left (this is, $T \in \mathcal{D}_{L}^{\prime}$ ), and assume that $D^{\mu} T$ is locally integrable for some $\mu>0$. Then, for any smooth function $\Phi$ on $\mathbb{R}, D^{\mu}(\Phi T)$ is locally integrable.

Of course, the analogous result holds for a distribution with compact support to the right and right derivatives $D_{\mu}$.

Let us also prove a result which is used in this paper, which says that the property that $D^{k} T$ is locally integrable is stronger the higher $k$ is:

Lemma 8.11. Let $T \in \mathcal{D}_{L}^{\prime}$ be a distribution on $\mathbb{R}$ with compact support to the left, and assume that $D^{k} T$ is locally integrable on $\mathbb{R}$ for some $k \in \mathbb{R}$. Then, $D^{k-m} T$ is locally integrable for all real $m \geq 0$.

Proof. It is enough to prove it for $k=0$, as then the general result is obtained by applying this particular case to the distribution $D^{k} T$, taking into account the composition law (8.12).

Then, to prove it for $k=0$, take $T$ a locally integrable function on $\mathbb{R}$ with support contained on $(R, \infty)$. Fix a compact interval $[a, b]$ with $b>R$. For a test function $\phi \in \mathcal{C}_{0}^{\infty}(\mathbb{R})$ with compact support contained on $(a, b)$ and any $m>0$ we have

$$
\left\langle D^{-m} T, \phi\right\rangle=\frac{1}{\Gamma(m)} \int_{R}^{b} T(y) \int_{y}^{b} \phi(z)(z-y)^{m-1} d z d y
$$


and hence

$$
\begin{aligned}
\left|\left\langle D^{-m} T, \phi\right\rangle\right| & \leq\|\phi\|_{\infty} \frac{1}{\Gamma(m)} \int_{R}^{b}|T(y)| \int_{y}^{b}(z-y)^{m-1} d z d y \\
& =\|\phi\|_{\infty} \frac{1}{\Gamma(m)} \int_{R}^{b}|T(y)| \int_{0}^{b-y} z^{m-1} d z d y \\
& \leq\|\phi\|_{\infty} \frac{(b-R)^{m}}{m \Gamma(m)} \int_{R}^{b}|T(y)| d y,
\end{aligned}
$$

which proves that $D^{-m} T$ is locally integrable on $(a, b)$.

\subsection{Regularity of a product by a smooth function}

In this section we give the proof of Theorem 8.10 on the regularity of fractional order of a product by a $\mathcal{C}^{\infty}$ function. We recall its statement:

Theorem 8.12. Let $T \in \mathcal{D}_{L}^{\prime}$ be a distribution on $\mathbb{R}$ with compact support to the left, and assume that $D^{\mu} T$ is locally integrable for some $\mu>0$. Then, for any smooth function $\Phi$ on $\mathbb{R}, D^{\mu}(\Phi T)$ is locally integrable.

The proof is broken into several lemmas. The following one sometimes serves as a weaker substitute for the rule of differentiation of a product:

Lemma 8.13. Take $0<k<1$. If $\phi, \psi \in \mathcal{C}^{\infty}(\mathbb{R})$ and have compact support to the right, then the following equality holds:

$$
\begin{aligned}
& D_{k}\left(\phi D_{-k} \psi\right)(y)= \\
& =\phi(y) \psi(y)-\frac{\sin (\pi k)}{\pi} \int_{y}^{\infty} \psi(x) \frac{1}{x-y} \int_{y}^{x} \phi^{\prime}(z)(x-z)^{k}(z-y)^{-k} d z d x .
\end{aligned}
$$

Proof. We have $D_{k}\left(\phi D_{-k} \psi\right)=D_{1} D_{k-1}\left(\phi D_{-k} \psi\right)$. Let us calculate this:

$$
\begin{aligned}
& D_{k-1}\left(\phi D_{-k} \psi\right)(y)= \\
& =\frac{1}{\Gamma(k) \Gamma(1-k)} \int_{y}^{\infty} \phi(z) \int_{z}^{\infty} \psi(x)(x-z)^{k-1}(z-y)^{-k} d x d z \\
& \quad=K_{1} \int_{y}^{\infty} \psi(x) \int_{y}^{x} \phi(z)(x-z)^{k-1}(z-y)^{-k} d z d x
\end{aligned}
$$

where we have set $K_{1}:=1 /(\Gamma(k) \Gamma(1-k))$ for short. By a change of variables $u=(z-y) /(x-y)$, the inner integral can be written as

$$
\int_{y}^{x} \phi(z)(x-z)^{k-1}(z-y)^{-k} d z=\int_{0}^{1} \phi(u(x-y)+y)(1-u)^{k-1} u^{-k} d u
$$


so we can calculate the derivative in $y$ of (8.17) and obtain

$$
\begin{aligned}
& D_{k}\left(\phi D_{-k} \psi\right)(y)=-K_{1} \frac{d}{d y} \int_{y}^{\infty} \psi(x) \int_{y}^{x} \phi(z)(x-z)^{k-1}(z-y)^{-k} d z d x \\
&=-K_{1} \frac{d}{d y} \int_{y}^{\infty} \psi(x) \int_{0}^{1} \phi(u(x-y)+y)(1-u)^{k-1} u^{-k} d u d x \\
&=\frac{B(k, 1-k)}{\Gamma(k) \Gamma(1-k)} \psi(y) \phi(y) \\
&-K_{1} \int_{y}^{\infty} \psi(x) \int_{0}^{1} \phi^{\prime}(u(x-y)+y)(1-u)^{k} u^{-k} d u d x \\
&= \psi(y) \phi(y)-K_{1} \int_{y}^{\infty} \psi(x) \frac{1}{x-y} \int_{0}^{1} \phi^{\prime}(z)(x-z)^{k}(z-y)^{-k} d z d x,
\end{aligned}
$$

where $B(k, 1-k)$ is the Beta function for the parameters $(k, 1-k)$. We have used the well-known relationship between the Beta and Gamma functions, and have undone our previous change of variables. This is the expression in the lemma; note that $K_{1}$ is the constant that appears there.

Lemma 8.14. Take $k \geq 0$ and $\Phi \in \mathcal{C}_{b}^{\infty}(\mathbb{R})$. Then, for all $\psi \in \mathcal{C}_{0}^{\infty}(\mathbb{R})$ with compact support contained on a fixed interval $(-\infty, b)$ it holds that

$$
\left|D_{-k}\left(\Phi D_{k-1} \psi\right)(y)\right| \leq(b-y)\|\psi\|_{\infty}\|\Phi\|_{\infty} \quad \text { for all } y<b .
$$

Proof. For $y \geq b, D_{-k}\left(\Phi D_{k-1} \psi\right)(y)$ is zero. For $y<b$ we have

$$
\begin{aligned}
\mid D_{-k}( & \left.\Phi D_{k-1} \psi\right)(y) \mid \leq \\
& \leq \frac{1}{\Gamma(k) \Gamma(1-k)} \int_{y}^{b}|\Phi(z)|(z-y)^{k-1} \int_{z}^{b}|\psi(x)|(x-z)^{-k} d x d z \\
& \leq \frac{1}{\Gamma(k) \Gamma(1-k)}\|\psi\|_{\infty}\|\Phi\|_{\infty} \int_{y}^{b} \int_{y}^{x}(z-y)^{k-1}(x-z)^{-k} d z d x \\
& \leq \frac{B(k, 1-k)}{\Gamma(k) \Gamma(1-k)}(b-y)\|\psi\|_{\infty}\|\Phi\|_{\infty}=(b-y)\|\psi\|_{\infty}\|\Phi\|_{\infty},
\end{aligned}
$$

taking into account the relationship between the Gamma and Beta functions.

Lemma 8.15. Take $\Phi \in \mathcal{C}_{b}^{\infty}(\mathbb{R})$ and $0 \leq k<1$. Fix $a<b \in \mathbb{R}$. Then, for all $\psi \in \mathcal{C}_{0}^{\infty}(\mathbb{R})$ with compact support contained on a fixed interval $(-\infty, b)$ it holds that

$$
\left\|D_{k}\left(\Phi D_{-k} \psi\right)\right\|_{L^{\infty}(a, b)} \leq K\|\psi\|_{\infty},
$$

where $K \geq 0$ is a constant that only depends on $k, \Phi$ and the interval $(a, b)$. 
Proof. For $k=0$ the statement is trivial. For $0<k<1$, we use Lemma 8.13 to write, for $y<b$,

$$
\begin{aligned}
D_{k}\left(\Phi D_{-k} \psi\right)(y) & =\Phi(y) \psi(y) \\
- & \frac{\sin (\pi k)}{\pi} \int_{y}^{b} \psi(x) \frac{1}{x-y} \int_{y}^{x} \Phi^{\prime}(z)(x-z)^{k}(z-y)^{-k} d z d x,
\end{aligned}
$$

where we have also taken into account that $\psi(x)$ is 0 for $x \geq b$. Here, the first term has the straightforward bound $\|\Phi \psi\|_{\infty} \leq\|\Phi\|_{\infty}\|\psi\|_{\infty}$. As for the second one,

$$
\begin{aligned}
\mid \int_{y}^{b} \psi(x) \frac{1}{x-y} & \int_{y}^{x} \Phi^{\prime}(z)(x-z)^{k}(z-y)^{-k} d z d x \mid \leq \\
& \leq\|\psi\|_{\infty}\left\|\Phi^{\prime}\right\|_{\infty} \int_{y}^{b} \frac{1}{x-y} \int_{y}^{x}(x-z)^{k}(z-y)^{-k} d z d x \\
& =K(b-y)\|\psi\|_{\infty}\left\|\Phi^{\prime}\right\|_{\infty},
\end{aligned}
$$

where the constant $K$ is $B(1+k, 1-k)$. Using this for $a<y<b$ proves the statement.

Lemma 8.16. Take $\Phi \in \mathcal{C}_{b}^{\infty}(\mathbb{R})$ and $k<1$. Fix $a<b \in \mathbb{R}$. Then, for all $\psi \in \mathcal{C}_{0}^{\infty}(\mathbb{R})$ with compact support contained on a fixed interval $(-\infty, b)$ it holds that

$$
\left\|D_{k}\left(\Phi D_{-k} \psi\right)\right\|_{L^{\infty}(a, b)} \leq K\|\psi\|_{\infty},
$$

where $K \geq 0$ is a constant that only depends on $k, \Phi$ and the interval $(a, b)$.

Proof. Lemma 8.15 proves this when $0 \leq k<1$, and we can prove the general case inductively: if the result is valid for a given $k<1$, then

$$
\begin{aligned}
D_{k-1}\left(\Phi D_{1-k} \psi\right) & =D_{k-1}\left(\Phi D_{1}\left(D_{-k} \psi\right)\right) \\
& =D_{k-1} D_{1}\left(\Phi D_{-k} \psi\right)-D_{k-1}\left(\left(D_{1} \Phi\right)\left(D_{-k} \psi\right)\right) \\
& =D_{k}\left(\Phi D_{-k} \psi\right)-D_{k-1}\left(\left(D_{1} \Phi\right)\left(D_{-k} \psi\right)\right) .
\end{aligned}
$$

For the first term we can use our induction hypothesis, and the second one can be bounded thanks to Lemma (8.18). This shows the lemma.

Proof of Theorem 8.10. Take $R \in \mathbb{R}$ so that $T$ has support contained in $(R, \infty)$. Fix a compact interval $(a, b)$ with $b>R$. For a function $\psi \in \mathcal{C}_{0}^{\infty}(\mathbb{R})$ with compact support on $(a, b)$ we have

$$
\begin{aligned}
\left\langle D^{\mu}(\Phi T), \psi\right\rangle=\left\langle\Phi T, D_{\mu} \psi\right\rangle & =\left\langle T, \Phi D_{\mu} \psi\right\rangle \\
& =\left\langle D^{-\mu} D^{\mu} T, \Phi D_{\mu} \psi\right\rangle=\left\langle D^{\mu} T, D_{-\mu} \Phi D_{\mu} \psi\right\rangle .
\end{aligned}
$$


Taking into account that $D^{\mu} T$ has support contained in $(R, \infty)$ and that $D_{-\mu} \Phi D_{\mu} \psi$ is smooth and has support contained in $(-\infty, b)$, we have

$\left|\left\langle D^{\mu}(\Phi T), \psi\right\rangle\right| \leq\left\|D^{\mu} T\right\|_{L^{1}(R, b)}\left\|D_{-\mu} \Phi D_{\mu} \psi\right\|_{L^{\infty}(R, b)} \leq K\left\|D^{\mu} T\right\|_{L^{1}(R, b)}\|\psi\|_{\infty}$,

thanks to Lemma 8.16.

\section{References}

[1] Aldous, D. J.: Deterministic and stochastic models for coalescence (aggregation, coagulation): a review of the mean-field theory for probabilists. Bernouilli 5 (1999), no. 1, 3-48.

[2] Cueille, S. and Sire, C.: Nontrivial polydispersity exponents in aggregation models. Phys. Rev. E 55 (1997), 5465-5478.

[3] Drake, R. L.: A general mathematical survey of the coagulation equation. In Topics in Current Aerosol Research (Part 2), volume 3, 201-376. International Reviews in Aerosol Physics and Chemistry. Pergamon, 1972.

[4] Escobedo, M. And Mischler, S.: Dust and self-similarity for the Smoluchowski coagulation equation. Ann. Inst. H. Poincaré Anal. Non Linéaire 23 (2006), no. 3, 331-362.

[5] Escobedo, M., Mischler, S. and Ricard, R. M.: On self-similarity and stationary problem for fragmentation and coagulation models. Ann. Inst. H. Poincaré Anal. Non Linéaire 22 (2005), no. 1, 99-125.

[6] Fournier, N. and Laurençot, P.: Existence of self-similar solutions to Smoluchowski's coagulation equation. Comm. Math. Phys. 256 (2005), no. 3, 589-609.

[7] Fournier, N. And LaurençOt, P.: Local properties of self-similar solutions to Smoluchowski's coagulation equation with sum kernels. Proc. Roy. Soc. Edinburgh Sect. A 136 (2006), no. 3, 485-508.

[8] Hartman, P.: Ordinary Differential Equations. Classics in Applied Mathematics 38. Society for Industrial and Applied Mathematics, Philadelphia, PA, 2002 .

[9] Kreer, M. and Penrose, O.: Proof of dynamical scaling in Smoluchowski's coagulation equation with constant kernel. J. Statist. Phys. 75 (1994), no. 3-4, 389-407.

[10] Laurençot, P. And Mischler, S.: On coalescence equations and related models. In Modeling and computational methods for kinetic equations, 321-356. Model. Simul. Sci. Eng. Technol. Birkhäuser, Boston, MA, 2004.

[11] Leyvraz, F.: Scaling theory and exactly solved models in the kinetics of irreversible aggregation. Physics Reports 383 (2003), no. 2-3, 95-212. 
[12] Menon, G. And Pego, R. L.: Approach to self-similarity in Smoluchowski's coagulation equations. Comm. Pure Appl. Math. 57 (2004), no. 9, $1197-1232$.

[13] Menon, G. and Pego, R. L.: Dynamical scaling in Smoluchowski's coagulation equations: uniform convergence. SIAM J. Math. Anal. 36 (2005), no. 5, 1629-1651.

[14] Oldham, K. B. And Spanier, J.: The fractional calculus; theory and applications of differentiation and integration to arbitrary order. Mathematics in Science and Engineering, V. Academic Press, 1974.

[15] Samko, S. G., Kilbas, A. A. and Marichev, O. I.: Fractional integrals and derivatives: Theory and applications. CRC, 1993.

[16] Schwartz, L.: Théorie des distributions. Hermann, 1997.

[17] Van Dongen, P. G. J. And Ernst, M. H.: Scaling solutions of Smoluchowski's coagulation equation. J. Statist. Phys. 50 (1988), no. 1-2, 295-329.

Recibido: 10 de noviembre de 2009

Revisado: 22 de abril de 2010

José A. Cañizo

Departament de Matemàtiques Universitat Autònoma de Barcelona 08193 Bellaterra (Barcelona), Spain canizo@mat.uab.cat

Stéphane Mischler CEREMADE, Univ. Paris-Dauphine Place du Maréchal de Lattre de Tassigny

75775 Paris CEDEX 16, France mischler@ceremade.dauphine.fr

The first author is supported by the project MTM2008-06349-C03-03 DGI-MICINN (Spain) and the French-Spanish project FR2009-0019. 